\title{
Measurement of dijet production in diffractive deep-inelastic $e p$ scattering at HERA
}

\section{H1 collaboration}

V. Andreev, ${ }^{21}$ A. Baghdasaryan, ${ }^{33}$ K. Begzsuren, ${ }^{30}$ A. Belousov, ${ }^{21}$ V. Boudry, ${ }^{24}$

G. Brandt, ${ }^{45}$ V. Brisson, ${ }^{23}$ D. Britzger, ${ }^{10}$ A. Buniatyan, ${ }^{2}$ A. Bylinkin, ${ }^{20,42}$

L. Bystritskaya, ${ }^{20}$ A.J. Campbell, ${ }^{10}$ K.B. Cantun Avila, ${ }^{19}$ F. Ceccopieri, ${ }^{3}$ K. Cerny, ${ }^{27}$

V. Chekelian, ${ }^{22}$ J.G. Contreras, ${ }^{19}$ J. Cvach, ${ }^{26}$ J.B. Dainton, ${ }^{16}$ K. Daum, ${ }^{32,37}$

C. Diaconu, ${ }^{18}$ M. Dobre, ${ }^{4}$ V. Dodonov, ${ }^{10}$ G. Eckerlin, ${ }^{10}$ S. Egli, ${ }^{31}$ E. Elsen, ${ }^{10}$

L. Favart, ${ }^{3}$ A. Fedotov, ${ }^{20}$ J. Feltesse, ${ }^{9}$ J. Ferencei, ${ }^{14}$ M. Fleischer, ${ }^{10}$ A. Fomenko, ${ }^{21}$

E. Gabathuler, ${ }^{16}$ J. Gayler, ${ }^{10}$ S. Ghazaryan, ${ }^{10}$ A. Glazov, ${ }^{10}$ L. Goerlich, ${ }^{6}$

N. Gogitidze, ${ }^{21}$ M. Gouzevitch, ${ }^{10,38}$ C. Grab, ${ }^{35}$ A. Grebenyuk, ${ }^{3}$ T. Greenshaw, ${ }^{16}$

G. Grindhammer, ${ }^{22}$ D. Haidt, ${ }^{10}$ R.C.W. Henderson, ${ }^{15}$ M. Herbst, ${ }^{13}$ J. Hladkỳ, ${ }^{26}$

D. Hoffmann, ${ }^{18}$ R. Horisberger, ${ }^{31}$ T. Hreus, ${ }^{3}$ F. Huber, ${ }^{12}$ M. Jacquet, ${ }^{23}$ X. Janssen, ${ }^{3}$

H. Jung, ${ }^{10,3}$ M. Kapichine, ${ }^{8}$ C. Kiesling, ${ }^{22}$ M. Klein, ${ }^{16}$ C. Kleinwort, ${ }^{10}$ R. Kogler, ${ }^{11}$

P. Kostka, ${ }^{16}$ J. Kretzschmar, ${ }^{16}$ K. Krüger, ${ }^{10}$ M.P.J. Landon, ${ }^{17}$ W. Lange, ${ }^{34}$

P. Laycock, ${ }^{16}$ A. Lebedev, ${ }^{21}$ S. Levonian, ${ }^{10}$ K. Lipka, ${ }^{10,41}$ B. List, ${ }^{10}$ J. List, ${ }^{10}$

B. Lobodzinski, ${ }^{22}$ E. Malinovski, ${ }^{21}$ H.-U. Martyn, ${ }^{1}$ S.J. Maxfield, ${ }^{16}$ A. Mehta, ${ }^{16}$

A.B. Meyer, ${ }^{10}$ H. Meyer, ${ }^{32}$ J. Meyer, ${ }^{10}$ S. Mikocki, ${ }^{6}$ A. Morozov, ${ }^{8}$ K. Müller, ${ }^{36}$

Th. Naumann, ${ }^{34}$ P.R. Newman, ${ }^{2}$ C. Niebuhr, ${ }^{10}$ G. Nowak, ${ }^{6}$ J.E. Olsson, ${ }^{10}$

D. Ozerov, ${ }^{10}$ P. Pahl, ${ }^{10}$ C. Pascaud, ${ }^{23}$ G.D. Patel, ${ }^{16}$ E. Perez, ${ }^{9,39}$ A. Petrukhin, ${ }^{10}$

I. Picuric, ${ }^{25}$ H. Pirumov, ${ }^{10}$ D. Pitzl, ${ }^{10}$ R. Plačakytė, ${ }^{10,41}$ B. Pokorny, ${ }^{27}$ R. Polifka, ${ }^{27,43}$

V. Radescu, ${ }^{10,41}$ N. Raicevic, ${ }^{25}$ T. Ravdandorj, ${ }^{30}$ P. Reimer, ${ }^{26}$ E. Rizvi, ${ }^{17}$

P. Robmann, ${ }^{36}$ R. Roosen, ${ }^{3}$ A. Rostovtsev, ${ }^{20}$ M. Rotaru, ${ }^{4}$ S. Rusakov, ${ }^{21}$ D. Šálek, ${ }^{27}$

D.P.C. Sankey, ${ }^{5}$ M. Sauter, ${ }^{12}$ E. Sauvan, ${ }^{18,44}$ S. Schmitt, ${ }^{10}$ L. Schoeffel, ${ }^{9}$

A. Schöning, ${ }^{12}$ H.-C. Schultz-Coulon, ${ }^{13}$ F. Sefkow, ${ }^{10}$ S. Shushkevich, ${ }^{10}$

Y. Soloviev, ${ }^{10,21}$ P. Sopicki, ${ }^{6}$ D. South, ${ }^{10}$ V. Spaskov, ${ }^{8}$ A. Specka, ${ }^{24}$ M. Steder, ${ }^{10}$

B. Stella, ${ }^{28}$ U. Straumann, ${ }^{36}$ T. Sykora, ${ }^{3,27}$ P.D. Thompson, ${ }^{2}$ D. Traynor, ${ }^{17}$ P. Truöl, ${ }^{36}$

I. Tsakov, ${ }^{29}$ B. Tseepeldorj, ${ }^{30,40}$ J. Turnau, ${ }^{6}$ A. Valkárová, ${ }^{27}$ C. Vallée, ${ }^{18}$

P. Van Mechelen, ${ }^{3}$ Y. Vazdik, ${ }^{21}$ D. Wegener, ${ }^{7}$ E. Wünsch, ${ }^{10}$ J. Žáček, ${ }^{27}$ Z. Zhang, ${ }^{23}$

R. Žlebčík, ${ }^{27}$ H. Zohrabyan ${ }^{33}$ and F. Zomer ${ }^{23}$

${ }^{1}$ I. Physikalisches Institut der RWTH, Aachen, Germany 
${ }^{2}$ School of Physics and Astronomy, University of Birmingham, Birmingham, U.K. ${ }^{\mathrm{a}}$

${ }^{3}$ Inter-University Institute for High Energies ULB-VUB, Brussels and Universiteit Antwerpen, Antwerpen, Belgium ${ }^{\mathrm{b}}$

${ }^{4}$ National Institute for Physics and Nuclear Engineering (NIPNE), Bucharest, Romaniac

${ }^{5}$ STFC, Rutherford Appleton Laboratory, Didcot, Oxfordshire, U.K. ${ }^{a}$

${ }^{6}$ Institute for Nuclear Physics, Cracow, Poland ${ }^{\mathrm{d}}$

${ }^{7}$ Institut für Physik, TU Dortmund, Dortmund, Germany ${ }^{\mathrm{e}}$

${ }^{8}$ Joint Institute for Nuclear Research, Dubna, Russia

${ }^{9} C E A, D S M / I r f u, C E-S a c l a y$, Gif-sur-Yvette, France

${ }^{10}$ DESY, Hamburg, Germany

${ }^{11}$ Institut für Experimentalphysik, Universität Hamburg, Hamburg, Germany ${ }^{e}$

${ }^{12}$ Physikalisches Institut, Universität Heidelberg, Heidelberg, Germany ${ }^{e}$

${ }^{13}$ Kirchhoff-Institut für Physik, Universität Heidelberg, Heidelberg, Germany ${ }^{e}$

${ }^{14}$ Institute of Experimental Physics, Slovak Academy of Sciences, Košice, Slovak Republic ${ }^{\mathrm{f}}$

${ }^{15}$ Department of Physics, University of Lancaster, Lancaster, U.K. ${ }^{a}$

${ }^{16}$ Department of Physics, University of Liverpool, Liverpool, U.K. ${ }^{a}$

${ }^{17}$ School of Physics and Astronomy, Queen Mary, University of London, London, U.K. ${ }^{a}$

${ }^{18}$ Aix Marseille Université, CNRS/IN2P3, CPPM UMR 7346, 13288 Marseille, France

${ }^{19}$ Departamento de Fisica Aplicada, CINVESTAV, Mérida, Yucatán, México ${ }^{g}$

${ }^{20}$ Institute for Theoretical and Experimental Physics, Moscow, Russia ${ }^{\mathrm{h}}$

${ }^{21}$ Lebedev Physical Institute, Moscow, Russia

${ }^{22}$ Max-Planck-Institut für Physik, München, Germany

${ }^{23}$ LAL, Université Paris-Sud, CNRS/IN2P3, Orsay, France

${ }^{24}$ LLR, Ecole Polytechnique, CNRS/IN2P3, Palaiseau, France

${ }^{25}$ Faculty of Science, University of Montenegro, Podgorica, Montenegro ${ }^{\mathrm{i}}$

${ }^{26}$ Institute of Physics, Academy of Sciences of the Czech Republic, Praha, Czech Republic ${ }^{\mathrm{j}}$

${ }^{27}$ Faculty of Mathematics and Physics, Charles University, Praha, Czech Republic ${ }^{j}$

${ }^{28}$ Dipartimento di Fisica Università di Roma Tre and INFN Roma 3, Roma, Italy

${ }^{29}$ Institute for Nuclear Research and Nuclear Energy, Sofia, Bulgaria

${ }^{30}$ Institute of Physics and Technology of the Mongolian Academy of Sciences, Ulaanbaatar, Mongolia

${ }^{31}$ Paul Scherrer Institut, Villigen, Switzerland

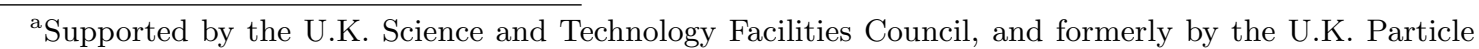
Physics and Astronomy Research Council.

${ }^{\mathrm{b}}$ Supported by FNRS-FWO-Vlaanderen, IISN-IIKW and IWT and by Interuniversity Attraction Poles Programme, Belgian Science Policy.

${ }^{\mathrm{c}}$ Supported by the Romanian National Authority for Scientific Research under the contract PN 09370101.

${ }^{\mathrm{d}}$ Partially Supported by Polish Ministry of Science and Higher Education, grant DPN/N168/DESY/2009.

${ }^{\text {e}}$ Supported by the Bundesministerium für Bildung und Forschung, FRG, under contract numbers 05H09GUF, 05H09VHC, 05H09VHF, 05H16PEA.

${ }^{\mathrm{f}}$ Supported by VEGA SR grant no. 2/7062/ 27.

${ }^{\mathrm{g}}$ Supported by CONACYT, México, grant 48778-F.

${ }^{\mathrm{h}}$ Russian Foundation for Basic Research (RFBR), grant no 1329.2008.2 and Rosatom.

${ }^{\mathrm{i}}$ Partially Supported by Ministry of Science of Montenegro, no. 05-1/3-3352.

${ }^{\mathrm{j}}$ Supported by the Ministry of Education of the Czech Republic under the projects LC527, INGOLA09042 and MSM0021620859. 
${ }^{32}$ Fachbereich C, Universität Wuppertal, Wuppertal, Germany

${ }^{33}$ Yerevan Physics Institute, Yerevan, Armenia

${ }^{34}$ DESY, Zeuthen, Germany

${ }^{35}$ Institut für Teilchenphysik, ETH, Zürich, Switzerland ${ }^{\mathrm{k}}$

${ }^{36}$ Physik-Institut der Universität Zürich, Zürich, Switzerland ${ }^{k}$

${ }^{37}$ Also at Rechenzentrum, Universität Wuppertal, Wuppertal, Germany.

${ }^{38}$ Also at IPNL, Université Claude Bernard Lyon 1, CNRS/IN2P3, Villeurbanne, France.

${ }^{39}$ Also at CERN, Geneva, Switzerland.

${ }^{40}$ Also at Ulaanbaatar University, Ulaanbaatar, Mongolia.

${ }^{41}$ Supported by the Initiative and Networking Fund of the Helmholtz Association (HGF) under the contract $V H-N G-401$ and S0-072.

${ }^{42}$ Also at Moscow Institute of Physics and Technology, Moscow, Russia.

${ }^{43}$ Also at Department of Physics, University of Toronto, Toronto, Ontario, Canada M5S $1 A \%$.

${ }^{44}$ Also at LAPP, Université de Savoie, CNRS/IN2P3, Annecy-le-Vieux, France.

${ }^{45}$ Department of Physics, Oxford University, Oxford, U.K. ${ }^{a}$

E-mail: daum@mail.desy.de

Abstract: A measurement is presented of single- and double-differential dijet cross sections in diffractive deep-inelastic ep scattering at HERA using data collected by the H1 experiment corresponding to an integrated luminosity of $290 \mathrm{pb}^{-1}$. The investigated phase space is spanned by the photon virtuality in the range of $4<Q^{2}<100 \mathrm{GeV}^{2}$ and by the fractional proton longitudinal momentum loss $x_{\mathbb{P}}<0.03$. The resulting cross sections are compared with next-to-leading order QCD predictions based on diffractive parton distribution functions and the value of the strong coupling constant is extracted.

KEYwORDS: Lepton-Nucleon Scattering, Diffraction, Jet physics

ArXiv EPrint: 1412.0928v2

\footnotetext{
${ }^{\mathrm{k}}$ Supported by the Swiss National Science Foundation.
} 


\section{Contents}

1 Introduction 1

2 Kinematics 2

3 Monte Carlo models and fixed order QCD calculations 3

4 Experimental technique $\quad 5$

4.1 H1 detector 5

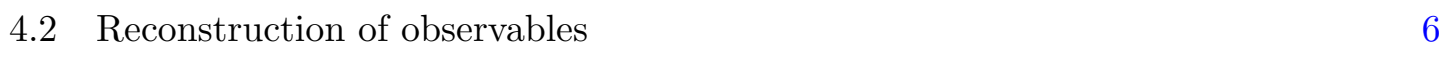

4.3 Event selection $\quad 7$

4.4 Corrections to the data 8

$\begin{array}{lll}4.5 & \text { Systematic uncertainties } & 9\end{array}$

$\begin{array}{llr}5 & \text { Results } & 10\end{array}$

6 Conclusions 12

\section{Introduction}

In deep-inelastic scattering (DIS), diffractive reactions of the type $e p \rightarrow e X Y$, where $X$ is a high-mass hadronic final state and $Y$ is either the elastically scattered proton or its low-mass excitation, represent about $10 \%$ of the events at HERA and provide rich experimental input for testing quantum chromodynamics (QCD) in the diffractive regime. These processes can be understood as probing by a virtual photon emitted from the beam lepton a net colour singlet carrying vacuum quantum numbers (a pomeron) [1, 2]. Due to the colourless exchange the systems $X$ and $Y$ are separated by a rapidity interval free of hadronic activities. In these processes at least one hard scale is involved such that perturbative QCD (pQCD) can be applied.

According to the QCD collinear factorisation theorem [3], calculations of diffractive cross sections factorise into process dependent hard scattering coefficient functions and a set of process independent diffractive parton distribution functions (DPDFs). While the hard scattering coefficient functions are calculable in $\mathrm{pQCD}$, the DPDFs have to be determined from QCD fits to the measured inclusive diffractive cross sections. In such QCD fits [4], DGLAP evolution [5-7] of the DPDFs is assumed. The QCD factorisation theorem is proven to hold for inclusive and dijet diffractive processes [8], assuming high enough photon virtuality such that higher twist effects can be neglected. The DPDFs are experimentally determined by assuming an additional factorisation of the DPDFs dependence on the scattered proton momentum from the dependence on the other variables, ascribed to the structure of the colourless exchange. This assumption is known as proton vertex 
factorisation. A pomeron flux in the proton is introduced and universal parton densities are attributed to the diffractively exchanged object. Many measurements of diffraction in DIS suggest the validity of the proton vertex factorisation assumption in DIS [4, 9-11].

In leading order the inclusive diffractive cross section in $e p$ scattering is proportional to the charge-squared weighted sum of the quark distribution functions in the pomeron, while its gluon content can be determined only indirectly via scaling violations. As events with two jets (dijets) are readily produced in gluon-induced processes, measurements of diffractive dijet cross sections are sensitive to the value of the strong coupling $\alpha_{s}$ and to the gluon content of the pomeron. The production of dijets in diffractive DIS has previously been studied at HERA using either the large rapidity gap (LRG) method [12-14] or by direct detection of the outgoing proton [15].

In this paper cross section measurements of dijet production in diffractive $e p$ scattering are presented, based on data collected in the years 2005-2007 with the H1 detector at HERA. Diffractive events are selected by means of the LRG method, requiring a clear separation in rapidity of the final state systems $X$ and $Y$. The measured cross sections are compared to next-to-leading order (NLO) QCD predictions evaluated with input DPDFs determined in previous inclusive diffractive measurements by the $\mathrm{H} 1$ collaboration [4].

The present analysis is based on the full HERA-II data sample resulting in significantly increased statistics with respect to previous analyses. Furthermore, the cross sections are determined using a regularised unfolding procedure which fully accounts for efficiencies, migrations and correlations among the measurements. The measured dijet cross sections are used to extract the strong coupling constant $\alpha_{s}$ in diffractive DIS processes for the first time.

\section{Kinematics}

A leading order (LO) diagram of boson-gluon fusion, which is the dominant process for the production of two jets in diffractive DIS, is depicted in figure 1. The incoming electron ${ }^{1}$ of four-momentum $k$ interacts with the incoming proton of four-momentum $p$ via the exchange of a virtual photon of four-momentum $q=k-k^{\prime}$. The outgoing proton or its low-mass dissociation state carries four-momentum $p^{\prime}$. The DIS kinematics is described by the following set of variables:

$$
Q^{2}=-q^{2}=\left(k-k^{\prime}\right)^{2}, \quad x=\frac{Q^{2}}{2 p \cdot q}, \quad y=\frac{p \cdot q}{p \cdot k},
$$

where $Q^{2}, x$ and $y$ denote the photon virtuality, the Bjorken- $x$ variable and the inelasticity of the process, respectively. Conservation laws stipulate the relation $Q^{2}=x y s$, where $s$ stands for the $e p$ centre-of-mass energy squared.

The kinematics of the diffractive exchange is described in terms of the additional quantities

$$
x_{\mathbb{P}}=\frac{q \cdot\left(p-p^{\prime}\right)}{q \cdot p}, \quad t=\left(p-p^{\prime}\right)^{2}
$$

\footnotetext{
${ }^{1}$ In this paper the term "electron" is used generically to refer to both electrons and positrons.
} 


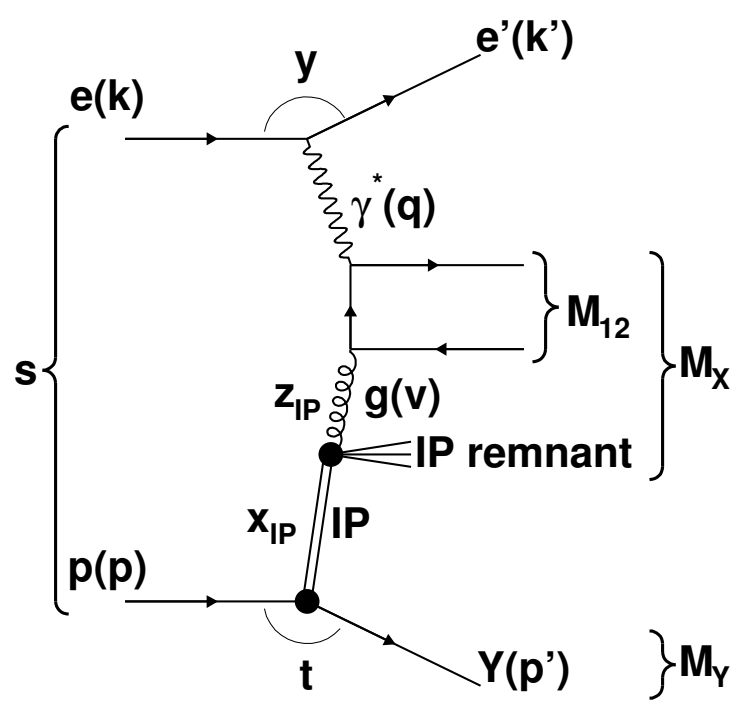

Figure 1: Leading order diagram for the production of dijets in diffractive DIS.

with $x_{\mathbb{P}}$ and $t$ being the longitudinal momentum fraction of the incoming proton carried by the pomeron and the squared four-momentum transfer at the proton vertex, respectively. The fractional longitudinal momentum of the pomeron transferred to the dijet system is given by

$$
z_{\mathbb{P}}=\frac{q \cdot v}{q \cdot\left(p-p^{\prime}\right)}=\frac{x}{x_{\mathbb{P}}},
$$

where $v$ is the four-momentum of the parton entering the hard interaction.

\section{Monte Carlo models and fixed order QCD calculations}

The RAPGAP event generator [16] allows for the simulation of processes ep $\rightarrow e X Y$ including both leading (pomeron) and sub-leading (reggeon) exchanges. Assuming the proton vertex factorisation, the parton densities obtained in the previous QCD analysis of inclusive diffractive data (H12006 Fit-B) [4] are convoluted with leading order QCD matrix elements. Higher order QCD radiation effects are modelled via initial and final state parton showers in the leading-log approximation [17]. Hadronisation is accounted for by making use of the Lund string model [18] as implemented in PYTHIA [19].

Within the diffractive selection based on the LRG method, the system $Y$ may also be a low mass dissociative system. Proton dissociation events are simulated in the the range of $M_{Y}<20 \mathrm{GeV}$ using the RAPGAP event generator, where $M_{Y}$ is the mass of the system $Y$. Resonant contributions together with the continuum part of the $M_{Y}$ distribution are modelled similarly to the DIFFVM event generator [20]. A small admixture of resolved $\gamma^{*} p$ scattering is included in fixed LO mode of jet production in the low $Q^{2}$ region [21]. The resolved photon contribution is simulated with the RAPGAP event generator using the SaS-G PDF set [22] as the input PDF of the photon. QED radiation effects are simulated with the HERACLES [23] program interfaced to RAPGAP. Besides the Born level contribution, the simulated cross sections include contributions from initial and final 
state emission of real photons from the electron, from vertex corrections as well as from self energy diagrams. As the H12006 Fit-B DPDF set has previously been observed to underestimate the data in the low $Q^{2}$ region, a weighting is applied for $Q^{2}<7 \mathrm{GeV}^{2}$, parametrised as the ratio of the data in [4] to the Monte Carlo expectation based on the H12006 Fit-B DPDF set.

Background arising from non-diffractive DIS processes is also simulated with the RAPGAP event generator using its inclusive mode together with the CTEQ6L PDF set [24].

The MC simulation is used to correct the data for detector effects. The generated events undergo the full GEANT [25] simulation of the H1 detector and are analysed in the same way as the real data. In order to describe the measured distributions, the diffractive MC is reweighted in several variables as discussed in 4.4.

QCD predictions of the dijet cross sections at the parton level are evaluated at NLO using the NLOJET ++ program $[26,27]$. The NLO pQCD predictions are calculated in the $\overline{\mathrm{MS}}$-scheme with five active flavors. The two-loop approximation of the renormalisation group equation is used for the running of the strong coupling constant with a coupling strength of $\alpha_{s}\left(M_{Z}\right)=0.118$. The cross sections are evaluated in intervals of $x_{\mathbb{P}}$, effectively replacing the beam proton by a pomeron (slicing method). The H12006 Fit-B DPDF set is used in the calculation. The renormalisation and factorisation scales $\mu_{r}$ and $\mu_{f}$ are provided by the photon virtuality and the average transverse momentum of the leading and sub-leading jet, $\left\langle p_{\mathrm{T}}^{*}\right\rangle$, in the $\gamma^{*}-p$ centre-of-mass frame and are defined as $\mu_{r}=\mu_{f}=\sqrt{\left\langle p_{\mathrm{T}}^{*}\right\rangle^{2}+Q^{2}}$. The uncertainty on the prediction due to missing higher orders is estimated by simultaneous variation of the renormalisation and factorisation scales by factors of 0.5 or 2 . An uncertainty on the NLO prediction from the experimental uncertainties on the DPDF set is obtained using the eigenvector decomposition of the uncertainties of the H12006 Fit-B DPDF set. This uncertainty is propagated to the NLO prediction using the sign-improved formulae for error propagation [28]. A significant contribution to the uncertainty of the H12006 Fit-B set originates from the restriction of the input data to $z_{\mathbb{P}}<0.8$ and the extrapolation of the DPDF to $z_{\mathbb{P}}>0.8$.

Whereas the measured cross sections are compared to the predictions obtained by the slicing method, an alternative method of adapting the NLO calculations for diffractive DIS is used in the $\alpha_{s}$ extraction. In order to provide theory predictions with different values of $\alpha_{s}\left(M_{Z}\right)$, the fastNLO method [29-31] is used. Cross section predictions are obtained by folding tabulated matrix elements obtained from NLOJET $++[26,27]$ with the DPDF parametrisation. The matrix elements are determined as a function of the observable of interest, the factorisation scale $\mu_{F}$ and the convolution variable $x$. The relation $x=x_{\mathbb{P}} z_{\mathbb{P}}$ is used when folding with the DPDF. This way predictions can be obtained for different choices of DPDFs, of $\alpha_{s}$ and of the renormalisation and the factorisation scales without having to calculate the matrix elements all over again. Settings identical to the slicing method are used for parameters such as renormalisation and factorisation scales or DPDF set and very good numerical agreement with the slicing method is found. The uncertainty on the prediction due to missing higher orders is estimated by varying the scales by a factor $f$, where $0.5<f<2$. 
Since the measured cross sections are given at the level of stable hadrons, the QCD predicted cross sections have to be corrected for effects of initial and final state parton showers, hadronisation and fragmentation. These corrections are determined for each of the measured cross sections as the ratio of hadron to parton level cross sections, predicted with the RAPGAP event generator. Two distinct models of parton showers, the leading-log approximation and the colour dipole model as implemented in the ARIADNE program [32], are used in this calculation. In each measurement interval the resulting correction is taken as the average of the values predicted by the two models and the uncertainties on the correction factors are taken as half the difference of the two predictions. The hadron level cross sections are on average about $5 \%$ higher than the parton level cross sections. The total uncertainty on the NLO QCD predictions is obtained as the quadratic sum of the uncertainties from scale variation, DPDF fit and hadronisation uncertainties.

\section{Experimental technique}

\subsection{H1 detector}

A detailed description of the detector can be found elsewhere [33]. Here only those detector components relevant for the present analysis are briefly described. A right-handed coordinate system with the origin at the nominal interaction point and with the $z$-axis pointing in the proton beam direction is conventionally chosen as the laboratory frame. The polar angle $\theta$ is measured with respect to the $z$-axis, while the direction in the $x-y$ plane is defined by the azimuthal angle $\phi$. The pseudorapidity is defined as $\eta=-\ln \tan (\theta / 2)$.

The liquid argon (LAr) sampling calorimeter [34] is located inside a $1.15 \mathrm{~T}$ solenoidal field and covers the polar angular range $4^{\circ}<\theta<154^{\circ}$. The energy resolutions for electromagnetic and hadronic showers as determined in test beam measurements [35, 36] are $\sigma(E) / E \propto 11 \% / \sqrt{E / \mathrm{GeV}} \oplus 1 \%$ and $\sigma(E) / E \propto 50 \% / \sqrt{E / \mathrm{GeV}} \oplus 2 \%$, respectively. The energy and scattering angle of the scattered electron is measured in a scintillating fibre calorimeter SpaCal [37,38] with a resolution of $\sigma(E) / E \propto 7 \% / \sqrt{E / \mathrm{GeV}} \oplus 1 \%$. The precision of the energy scale is $1 \%$ covering the polar angular range $154^{\circ}<\theta_{e^{\prime}}<174^{\circ}$. The measurement of the polar angle of the scattered electron $\theta_{e^{\prime}}$ is improved by means of a backward proportional chamber (BPC). The precision of the polar angle measurement is $1 \mathrm{mrad}$.

Trajectories of charged particles are measured with the central tracking detector (CTD) located inside the LAr calorimeter with a transverse momentum resolution of $\sigma_{p_{T}} / p_{T} \simeq$ $0.2 \% \cdot p_{T} / \mathrm{GeV} \oplus 1.5 \%$ in the polar angular range of $15^{\circ}<\theta<165^{\circ}$.

The information from CTD and LAr is used for the reconstruction of the system $X$. The interaction vertex position is determined event-by-event using the particle trajectories measured in CTD.

The following $\mathrm{H} 1$ forward detectors are used in the LRG selection of diffractive events. The forward muon detector (FMD) consists of six proportional chambers which are grouped into two three-layer sections separated by a toroidal magnet. Although the nominal coverage of FMD is $1.9<\eta<3.7$, particles with pseudorapidity up to $\eta \sim 6.5$ can be detected indirectly through their interactions with the beam transport system and detector support 
structures. The lead-scintillator Plug calorimeter is located at $z=4.9 \mathrm{~m}$ and covers the range $3.5<\eta<5.5$. The very forward region is covered by the forward tagging system (FTS) comprising scintillators surrounding the beam pipe. Only one station of FTS, situated at $z=28 \mathrm{~m}$ and covering the range $6.0<\eta<7.5$, is included in the present analysis.

The instantaneous luminosity is monitored based on the rate of the Bethe-Heitler process $e p \rightarrow e p \gamma$. The final state photon is detected by a photon detector located close to the beam pipe at $z=-103 \mathrm{~m}$. The precision of the integrated luminosity measurement is improved in a dedicated analysis of the QED Compton process [39].

\subsection{Reconstruction of observables}

The DIS observables $Q^{2}, x$ and $y$ are reconstructed using the electron- $\Sigma$ method [40]. Within this method, the photon virtuality $Q^{2}$ is reconstructed based on the measured four-momentum of the scattered electron, while the inelasticity $y$ and Bjorken- $x$ are determined making use of combined information from the hadronic final state (HFS) and the scattered electron.

The four-momenta of the particles attributed to HFS are reconstructed using an algorithm which combines information provided by the tracking system and the LAr calorimeter by avoiding double counting of hadronic energy [41, 42]. The calibration of the HFS energy scale derived in [43] is applied. The performance of the calibration was studied by comparing the transverse momentum balance in data and $\mathrm{MC}$ in the kinematic domain of this analysis.

Jets are reconstructed in the $\gamma^{*}-p$ centre-of-mass frame using the inclusive $k_{\mathrm{T}}$ jet algorithm [44] with the $p_{\mathrm{T}}$ recombination scheme as implemented in the FastJet program [45]. The jet distance parameter is set to $R=1.0$. The transverse momenta and pseudorapidities of the leading and sub-leading jets are denoted as $p_{\mathrm{T}, 1}^{*}, \eta_{1}^{*}$ and $p_{\mathrm{T}, 2}^{*}, \eta_{2}^{*}$, respectively. ${ }^{2}$ The invariant mass of the final state system $X$ is reconstructed as:

$$
M_{X}=c\left(\eta_{\max }\right) \sqrt{P_{X}^{2}},
$$

where $P_{X}$ is the four-momentum of the system $X$ obtained as a vector sum of all particles contained in the HFS. The MC simulation is used in order to derive the average correction for detector losses $c\left(\eta_{\max }\right)$, where $\eta_{\max }$ is the pseudorapidity of the most forward energy deposition above $800 \mathrm{MeV}$ in the LAr calorimeter. The momentum fractions $x_{\mathbb{P}}$ and $z_{\mathbb{P}}$ are reconstructed as:

$$
x_{\mathbb{P}}=\frac{Q^{2}+M_{X}^{2}}{y s}
$$

and

$$
z_{\mathbb{P}}=\frac{Q^{2}+M_{12}^{2}}{Q^{2}+M_{X}^{2}}
$$

where $M_{12}$ is the invariant mass of the dijet system.

Cross sections for dijet production in diffractive DIS are measured differentially with respect to the variables $Q^{2}, y, x_{\mathbb{P}}, z_{\mathbb{P}}, p_{\mathrm{T}, 1}^{*}, p_{\mathrm{T}, 2}^{*},\left\langle p_{\mathrm{T}}^{*}\right\rangle=\left(p_{\mathrm{T}, 1}^{*}+p_{\mathrm{T}, 2}^{*}\right) / 2$ and $\Delta \eta^{*}=\left|\eta_{1}^{*}-\eta_{2}^{*}\right|$.

\footnotetext{
${ }^{2}$ Observables in the $\gamma^{*}-p$ centre-of-mass frame are labelled with an asterisk.
} 


\subsection{Event selection}

The measurement is based on the $\mathrm{H} 1$ data collected in the years 2005 to 2007 with a total integrated luminosity of $290 \mathrm{pb}^{-1}$. The nominal beam energies of the protons and electrons are $E_{p}=920 \mathrm{GeV}$ and $E_{e}=27.6 \mathrm{GeV}$, respectively.

The longitudinal position of the reconstructed event vertex is restricted to the range $-35<z_{\mathrm{vtx}}<35 \mathrm{~cm}$. DIS events are selected by the identification of the scattered electron in the backward calorimeter SpaCal. The isolated energy deposit of electromagnetic structure with the highest transverse momentum is identified as scattered electron and has to have a measured energy of at least $9.5 \mathrm{GeV}$.

Only events accepted by a trigger combining signals induced by the scattered electron in the SpaCal with minimum track information of the CTD are used in the analysis. The trigger efficiency related to the CTD condition is found to be $98 \%-99 \%$, depending on the detector configuration and is reproduced by the MC simulation within $2 \%$. The trigger efficiency related to the SPACAL condition is better than $99 \%$.

Residual non-DIS background is dominated by photoproduction processes, where a hadron is misidentified as the scattered electron, whereas the true scattered electron escapes detection due to its small scattering angle. This background is reduced to a negligible level by demanding $35<\sum_{i}\left(E-p_{z}\right)_{i}<75 \mathrm{GeV}$, where the sum runs over all HFS particles and the scattered electron candidate. Elastic QED Compton scattering $e p \rightarrow e \gamma p$ introduces another background contribution which is suppressed by rejecting configurations with two back-to-back clusters in SpaCal.

Diffractive events are identified with the LRG method which requires an empty interval in rapidity between the systems $X$ and $Y$. The low-mass system $Y$ is produced at very large pseudorapidities and escapes detection. The diffractive signature is thus defined by the systems $X$ (in the main detector) and $Y$ (undetected). The energy of any cluster in the forward region of the LAr calorimeter is required to be below the noise level of $800 \mathrm{MeV}$, which is ensured by demanding $\eta_{\max }<3.2$. The variable $\eta_{\max }$ corresponds to the LAr cluster above the noise threshold which has the largest pseudorapidity. Information provided by the forward detectors FMD, FTS and the Plug calorimeter is used in order to extend the gap to rapidities beyond the LAr acceptance and in order to suppress the proton dissociation contribution. These detectors are required to show no signal above noise level [46]. At high momentum fractions $x_{\mathbb{P}}$, the system $X$ tends to extend into the direction of the outgoing system $Y$ and the experimental separation of the systems $X$ and $Y$ is not possible. The LRG selection method is thus applicable only in the region of $x_{\mathbb{P}} \lesssim 0.03$. The sample of DIS events satisfying the LRG criteria is dominated by the diffractive exchange, as the system $X$ is isolated in the main part of the $\mathrm{H} 1$ detector, while the system $Y$ escapes undetected down the beam pipe. The signal is dominated by protonelastic processes, $e p \rightarrow e X p$, however, a small fraction of proton dissociation events is also accepted by the LRG selection. The LRG requirements impose restrictions on the mass and scattering angle of the hadronic system $Y$. These correspond approximately to the requirements $M_{Y}<1.6 \mathrm{GeV}$ and $|t|<1 \mathrm{GeV}^{2}$. Migrations in these variables are modelled using MC simulations. 


\begin{tabular}{|c|c|c|}
\hline & Extended Analysis Phase Space & Measurement Cross section Phase Space \\
\hline \multirow{2}{*}{ DIS } & $3<Q^{2}<100 \mathrm{GeV}^{2}$ & $4<Q^{2}<100 \mathrm{GeV}^{2}$ \\
& $y<0.7$ & $0.1<y<0.7$ \\
\hline \multirow{3}{*}{ Diffraction } & $x_{\mathbb{P}}<0.04$ & $x_{\mathbb{P}}<0.03$ \\
& LRG requirements & $|t|<1 \mathrm{GeV}^{2}$ \\
& & $M_{Y}<1.6 \mathrm{GeV}$ \\
\hline \multirow{3}{*}{ Dijets } & $p_{\mathrm{T}, 1}^{*}>3.0 \mathrm{GeV}$ & $p_{\mathrm{T}, 1}^{*}>5.5 \mathrm{GeV}$ \\
& $p_{\mathrm{T}, 2}^{*}>3.0 \mathrm{GeV}$ & $p_{\mathrm{T}, 2}^{*}>4.0 \mathrm{GeV}$ \\
& $-2<\eta_{1,2}^{\text {lab }}<2$ & $-1<\eta_{1,2}^{\text {lab }}<2$ \\
\hline
\end{tabular}

Table 1: Summary of the extended analysis phase space and the phase space for the dijet cross sections measurements.

Events are selected in a phase space which is extended compared to the measurement phase space in order to improve the precision of the measurement by accounting for migrations at the phase space boundaries. Events within the DIS phase space of $y<0.7$ and $3<Q^{2}<100 \mathrm{GeV}^{2}$ are selected. The events are required to have at least two jets in the pseudorapidity range $-2<\eta_{1,2}^{\text {lab }}<2$ and transverse momenta greater than $3 \mathrm{GeV}$ in the $\gamma^{*}-p$ centre-of-mass frame.

The measurement phase is defined by the DIS requirements of $0.1<y<0.7$ and $4<Q^{2}<100 \mathrm{GeV}^{2}$. The pseudorapidity of jets is restricted in the laboratory frame to $-1<\eta_{1,2}^{\mathrm{lab}}<2$ to ensure the jets to be contained well within the central detector. The transverse momenta of the leading and sub-leading jets are required to be larger than $5.5 \mathrm{GeV}$ and $4.0 \mathrm{GeV}$, respectively. The extended phase space and the measurement phase space definitions are summarised in table 1. The total number of events accepted by the LRG selection criteria together with the DIS and jet requirements is $\sim 50000$ and $\sim 15000$ for the extended and measurement phase space, respectively.

\subsection{Corrections to the data}

Cross sections at the level of stable hadrons are obtained from the measured event rates in data by applying corrections determined using the MC simulation. In figure 2 kinematic distributions of the observables $Q^{2}, p_{\mathrm{T}, 1}^{*}, x_{\mathbb{P}}$ and $z_{\mathbb{P}}$ as observed in the detector are shown in comparison to the expectations from the reweighted MC simulation. The overall good description of the data is achieved after applying a dedicated weighting of the MC simulation in the variables $z_{\mathbb{P}}, x_{\mathbb{P}}$ and $x_{\text {dijet }}=\sum_{1,2}\left(E^{* \text { jet }}-p_{z}^{* \text { jet }}\right)_{i} / \sum_{\mathrm{HFS}}\left(E^{*}-p_{z}^{*}\right)_{i}$. Weights are obtained from the reconstructed kinematic distributions and are applied at the hadron level. This procedure is iterated until a good description of the shapes of the observables is achieved.

The data are corrected for detector inefficiencies, acceptance and finite resolution using the regularised unfolding procedure as implemented in TUnfold [47]. A detector response matrix $A$, with elements $a_{i j}$ expressing the probability for an observable originating in the generated MC sample from an interval $i$ to be measured in an interval $j$, is determined using 
the MC simulation. Migrations from outside the measurement phase space are included by additional rows of the detector response matrix. The domains of jets with $3.0<p_{\mathrm{T}, 1}^{*}<$ $5.5 \mathrm{GeV}$ and of events with $0.03<x_{\mathbb{P}}<0.04$ are found to be the dominating sources of these migrations. The MC simulation is reweighted in order to describe the data also in these regions beyond the nominal phase space.

Two sources of background are considered in this analysis and are subtracted from the data using Monte Carlo simulations prior to unfolding: diffractive dijet events with $M_{Y}>1.6 \mathrm{GeV}$ and $|t|<1 \mathrm{GeV}^{2}$ and background from non-diffractive DIS.

For a background subtracted measurement $y_{j}$, the corresponding number of events in the truth bin $i, x_{i}$, is found by solving a minimisation problem for a $\chi^{2}$ function

$$
\chi^{2}=(y-A x)^{T} V_{y y}^{-1}(y-A x)+\tau^{2} x^{2},
$$

where $x$ and $y$ are vectors defined by $y_{j}$ and $x_{i}$, respectively, $V_{y y}$ is the covariance matrix accounting for the statistical uncertainties of $y_{j}$ and $\tau$ is a regularisation parameter introduced in order to damp statistical fluctuations of the solution. The regularisation parameter $\tau$ is determined using the L-Curve scan [47].

The cross section in each measurement interval $i$ is given by

$$
\sigma_{i}\left(e p \rightarrow e p^{\prime} X\right)=\frac{x_{i}}{\mathcal{L}}\left(1+\delta_{i, \mathrm{rad}}\right)
$$

where $\mathcal{L}$ is the integrated luminosity of the data sample and $\left(1+\delta_{i, \text { rad }}\right)$ is the correction for QED radiation effects in the interval $i$. These corrections are calculated as a ratio of RAPGAP predictions with and without QED radiation simulated. The differential cross section is determined by dividing $\sigma_{i}$ by the area of the corresponding interval.

\subsection{Systematic uncertainties}

The systematic uncertainties induced by experimental effects and by the process modelling are propagated to each measurement interval in the unfolding procedure (eq. (4.4)). A dedicated detector response matrix is constructed for each variation related to particular sources of uncertainties:

- The energy of the scattered electron is varied by $\pm 1 \%$ with a resulting uncertainty on the integrated dijet cross section of $1 \%$.

- The polar angle of the scattered electron is varied by $\pm 1 \mathrm{mrad}$ with a resulting uncertainty on the integrated dijet cross section of $1 \%$.

- The energy of each particle contained in HFS is varied by $\pm 1 \%$ [43] which translates into an uncertainty on the integrated dijet cross section of $4 \%$.

- Uncertainties related to the model dependent corrections of the data are accounted for by varying the shape of the kinematic distributions in $Q^{2}, x_{\mathbb{P}}, \beta, p_{\mathrm{T}, 1}^{*}, z_{\mathbb{P}}$, $x_{\text {dijet }}$ and $\Delta \eta^{*}$ in the MC such that the data are still described within the statistical uncertainties. For this purpose, the multiplicative weights $\left(\log Q^{2}\right)^{ \pm 0.2}, x_{\mathbb{P}}^{ \pm 0.05}$, 
$\beta^{ \pm 0.01}\left(1-\beta^{ \pm 0.01}\right), p_{\mathrm{T}, 1}^{* \pm 0.04}, z_{\mathbb{P}}^{ \pm 0.15}, x_{\mathrm{dijet}}^{ \pm 0.15}$ and $\left(1.5+\Delta \eta^{*}\right)^{ \pm 0.5}$ are applied, respectively. The largest resulting uncertainty of $3 \%$ arises from the variation of the shape in $p_{\mathrm{T}, 1}^{*}$. The shape of the distribution in $t$ is varied within the experimental uncertainty on the $t$-slope [48] by applying a weight of $e^{ \pm t}$ in $\mathrm{MC}$, which translates into an uncertainty on the integrated dijet cross section of $1 \%$. The integrated cross section uncertainty due to the model dependence of the measurement is of the order of $5 \%$.

The following uncertainties on the global normalisation are considered:

- The luminosity of the data is measured with a precision $\pm 2.7 \%$ [39].

- The trigger efficiency related to the tracking and SpaCal condition induces an uncertainty of $2 \%$ and $1 \%$, respectively.

- The uncertainty accounting for the LRG selection efficiency is 7\% [49].

- The normalisation of the non-diffractive DIS background modelled by RAPGAP is varied by $\pm 50 \%$ and the normalisation of the diffractive background is varied by $\pm 100 \%$, yielding a resulting uncertainty on the integrated dijet cross section below $1 \%$ in both cases.

The total systematic uncertainty is obtained by adding the individual contributions in quadrature.

\section{Results}

The integrated cross section in the measurement phase space specified in table 1 is found to be

$$
\sigma_{\text {meas }}^{\text {dijet }}(e p \rightarrow e X Y)=73 \pm 2 \text { (stat.) } \pm 7 \text { (syst.) pb . }
$$

The NLO QCD prediction of the total diffractive dijet cross section is

$$
\sigma_{\text {theo }}^{\text {dijet }}(e p \rightarrow e X Y)=77_{-20}^{+25}(\text { scale }){ }_{-14}^{+4}(\mathrm{DPDF}) \pm 3(\text { had }) \mathrm{pb},
$$

in very good agreement with the measurement. The uncertainty on the NLO prediction is found to be significantly larger than the experimental uncertainty.

Single differential cross sections are given in tables 2 and 3 and are shown in figures 3-6. The statistical correlations between measurements in different bins are given in tables 6 and 7. The differential cross sections as a function of the DIS variables $Q^{2}$ and $y$ are shown in figure 3 , as a function of the momentum fractions $x_{\mathbb{P}}$ and $z_{\mathbb{P}}$ are shown in figure 4 and as a function of the jet variables $p_{T, 1}^{*}, p_{T, 2}^{*},\left\langle p_{T}^{*}\right\rangle$ and $\Delta \eta^{*}$ are shown in figure 5 and 6 . For the majority of the measurements, the data precision is limited by systematic effects. The statistical correlations are small for the inclusive kinematic variables $Q^{2}$ and $y$ and moderate $(|\rho|<0.6)$ for the other variables. The figures also include the NLO QCD predictions which describe within their large uncertainties the data well.

The dynamics of dijet production is further studied in terms of double differential cross sections in bins of $z_{\mathbb{P}}$ and of the QCD scale defining observables $Q^{2}$ and $p_{\mathrm{T}, 1}^{*}$. The 
double differential cross sections are listed in tables $4-5$ and are shown in figures $7-10$. The corresponding statistical correlations between measurements in different bins are given in tables 8-9. Figure 7 shows the double differential cross section measured in bins of $z_{\mathbb{P}}$ and $Q^{2}$. The ratio of the data to the theory prediction is shown in figure 8 . The data are well described by the NLO prediction in most of the phase space. The double differential cross section measured in bins of $p_{\mathrm{T}, 1}^{*}$ and $Q^{2}$ is shown in figure 9 and the corresponding ratios of the measurements to the NLO predictions are shown in figure 10.

The present measurement is based on a six times increased luminosity as compared to the previous H1 measurement of dijet production with LRG [13] and is using a more sophisticated data correction method. A direct comparison of the present data to other measurements of dijet production in diffractive DIS is not possible because of different phase space definitions. Measurements based on the direct detection of a forward proton [15] are limited in statistical precision due to the restricted geometrical acceptance of the proton taggers.

The experimental uncertainties on both single- and double-differential cross sections are in general smaller than the theory uncertainties. The data thus have the power to constrain QCD in diffractive DIS. Here, the double-differential dijet cross sections as a function of $Q^{2}$ and $p_{\mathrm{T}, 1}^{*}$ are used to determine the value of the strong coupling constant $\alpha_{s}\left(M_{Z}\right)$ at the scale of the mass of the $Z$-boson, $M_{Z}$. The value of $\alpha_{s}\left(M_{Z}\right)$ is determined by an iterative $\chi^{2}$-minimisation procedure using NLO calculations, corrected for hadronisation effects following the method [50]. In the fit, the uncertainties on the HFS energy scale are treated as 50\% correlated and 50\% uncorrelated. All other experimental uncertainties are treated as correlated. Scale uncertainties, hadronisation uncertainties and DPDF uncertainties of the NLO calculation are propagated to the fit result as described in [50].

The fit yields a value of $\chi^{2} / n_{\text {dof }}=16.7 / 14$, with $n_{\text {dof }}$ being the number of degrees of freedom, thus indicating good agreement of theory to data. The nuisance parameters of the correlated systematic uncertainties are equally distributed around zero with absolute values below one. The value of $\alpha_{s}\left(M_{Z}\right)$ determined in the fit to the dijet cross sections is

$$
\begin{aligned}
\alpha_{s}\left(M_{Z}\right) & =0.119 \pm 0.004(\exp ) \pm 0.002(\mathrm{had}) \pm 0.005(\mathrm{DPDF}) \pm 0.010\left(\mu_{r}\right) \pm 0.004\left(\mu_{f}\right) \\
& =0.119 \pm 0.004(\exp ) \pm 0.012(\mathrm{DPDF}, \text { theo })
\end{aligned}
$$

The largest uncertainties arise from the estimate of the contributions from orders beyond NLO and from the poor knowledge of the DPDF. The largest contribution to the experimental uncertainty of 0.003 arises from the global normalisation uncertainty.

The result for $\alpha_{s}\left(M_{Z}\right)$ is consistent within the uncertainties with the world average [51, $52]$ and with values from other jet data in DIS and photoproduction [50, 53, 54] as well as values of $\alpha_{s}\left(M_{Z}\right)$ determined from jet data at the Tevatron $[55,56]$ and at the LHC $[57,58]$. Although the uncertainty of this $\alpha_{s}\left(M_{Z}\right)$ extraction is not competitive with measurements in other processes the agreement with the other measurements supports the underlying concept of treating dijet production in diffractive DIS with perturbative QCD calculations. 


\section{Conclusions}

Integrated, single- and double-differential cross sections of diffractive DIS dijet production are measured with the $\mathrm{H} 1$ experiment in ep collisions at HERA and compared with NLO QCD predictions.

The integrated diffractive dijet cross section is found to be well described by the NLO QCD prediction using the H12006 Fit-B DPDF set. Both shapes and normalisation of the single-differential cross sections are reproduced by the theory within the experimental and theory uncertainties, confirming at improved precision the conclusions from previous H1 measurements. Good agreement of the theory with the measurement is also found for the shapes and normalisation of the double differential cross sections. The cross section measurements presented here show experimental uncertainties significantly smaller than the uncertainties of the theory predictions. From a fit of the NLO prediction to the double differential cross sections in $Q^{2}$ and $p_{T, 1}^{*}$, the strong coupling constant has been determined to be $\alpha_{s}\left(M_{Z}\right)=0.119(4)_{\exp }(12)_{\text {theo }}$.

\section{Acknowledgments}

We are grateful to the HERA machine group whose outstanding efforts have made this experiment possible. We thank the engineers and technicians for their work in constructing and maintaining the $\mathrm{H} 1$ detector, our funding agencies for financial support, the DESY technical staff for continual assistance and the DESY directorate for the hospitality which they extend to the non-DESY members of the collaboration. We would like to give credit to all partners contributing to the EGI computing infrastructure for their support for the

H1 Collaboration. 


\begin{tabular}{|c|c|c|c|c|c|c|c|c|c|c|c|c|c|c|c|c|c|c|}
\hline $\begin{array}{c}Q^{2} \\
{\left[\mathrm{GeV}^{2}\right]}\end{array}$ & $\begin{array}{c}d \sigma / d Q^{2} \\
{\left[\mathrm{pb} / \mathrm{GeV}^{2}\right]}\end{array}$ & $\begin{array}{l}\delta_{\text {tot }} \\
{[\%]}\end{array}$ & $\begin{array}{c}\delta_{\text {stat }} \\
{[\%]}\end{array}$ & $\begin{array}{l}\delta_{\text {sys }} \\
{[\%]}\end{array}$ & $\begin{array}{c}\delta_{\theta} \\
{[\%]}\end{array}$ & $\begin{array}{c}\delta_{E} \\
{[\%]}\end{array}$ & $\begin{array}{c}\delta_{\mathrm{HFS}} \\
{[\%]} \\
\end{array}$ & $\begin{array}{l}\delta_{Q^{2}} \\
{[\%]}\end{array}$ & $\begin{array}{l}\delta_{x_{P}} \\
{[\%]}\end{array}$ & $\begin{array}{c}\delta_{\beta} \\
{[\%]}\end{array}$ & $\begin{array}{c}\delta_{p *_{T, 1}} \\
{[\%]}\end{array}$ & $\begin{array}{l}\delta_{z_{\mathbb{P}}} \\
{[\%]}\end{array}$ & $\begin{array}{c}\delta_{x_{\text {dijet }}} \\
{[\%]}\end{array}$ & $\begin{array}{c}\delta_{\Delta \eta *} \\
{[\%]}\end{array}$ & $\begin{array}{c}\delta_{t} \\
{[\%]}\end{array}$ & $\begin{array}{l}\delta_{\mathrm{bgr}} \\
{[\%]}\end{array}$ & $1+\delta_{\text {had }}$ & $1+\delta_{\mathrm{rad}}$ \\
\hline $4 \div 6$ & 8.20 & 13.2 & 5.7 & 11.9 & 1.0 & 4.5 & -3.9 & 2.1 & 1.1 & 2.8 & -5.0 & 1.9 & 0.7 & 1.2 & -0.9 & 0.1 & $1.05 \pm 0.05$ & 1.05 \\
\hline $6 \div 10$ & 4.23 & 11.8 & 4.0 & 11.0 & 2.6 & 1.7 & -5.0 & -0.5 & 0.5 & -0.5 & -3.1 & -3.2 & -1.6 & 1.6 & -1.5 & 0.3 & $1.05 \pm 0.04$ & 1.03 \\
\hline $10 \div 18$ & 1.92 & 11.4 & 4.0 & 10.7 & 1.0 & 1.9 & -4.6 & -0.9 & 0.6 & -0.8 & -3.1 & -3.1 & -1.5 & 1.7 & -0.9 & 0.4 & $1.05 \pm 0.04$ & 1.03 \\
\hline $18 \div 34$ & 0.797 & 11.6 & 4.8 & 10.5 & 1.1 & 2.1 & -5.1 & 0.1 & 0.6 & -0.1 & -2.9 & -2.5 & -1.3 & 1.4 & -0.6 & 0.2 & $1.06 \pm 0.04$ & 1.03 \\
\hline $34 \div 100$ & 0.164 & 12.3 & 6.2 & 10.6 & 0.9 & 2.3 & -5.0 & -0.2 & 0.5 & -0.6 & -2.7 & -2.9 & -1.5 & 1.6 & -0.8 & 0.1 & $1.06 \pm 0.04$ & 1.03 \\
\hline$y$ & $\begin{array}{c}d \sigma / d y \\
{[\mathrm{pb}]}\end{array}$ & $\begin{array}{l}\delta_{\text {tot }} \\
{[\%]}\end{array}$ & $\begin{array}{c}\delta_{\text {stat }} \\
{[\%]}\end{array}$ & $\begin{array}{l}\delta_{\text {sys }} \\
{[\%]}\end{array}$ & $\begin{array}{c}\delta_{\theta} \\
{[\%]}\end{array}$ & $\begin{array}{c}\delta_{E} \\
{[\%]}\end{array}$ & $\begin{array}{c}\delta_{\mathrm{HFS}} \\
{[\%]}\end{array}$ & $\begin{array}{l}\delta_{Q^{2}} \\
{[\%]}\end{array}$ & $\begin{array}{l}\delta_{x_{\mathbb{P}}} \\
{[\%]}\end{array}$ & $\begin{array}{c}\delta_{\beta} \\
{[\%]}\end{array}$ & $\begin{array}{c}\delta_{p *_{T, 1}} \\
{[\%]}\end{array}$ & $\begin{array}{l}\delta_{z_{\mathbb{P}}} \\
{[\%]}\end{array}$ & $\begin{array}{c}\delta_{x_{\text {dijet }}} \\
{[\%]}\end{array}$ & $\begin{array}{c}\delta_{\Delta \eta *} \\
{[\%]}\end{array}$ & $\begin{array}{c}\delta_{t} \\
{[\%]}\end{array}$ & $\begin{array}{l}\delta_{\mathrm{bgr}} \\
{[\%]}\end{array}$ & $1+\delta_{\text {had }}$ & $1+\delta_{\mathrm{rad}}$ \\
\hline $0.10 \div 0.22$ & 113 & 18.4 & 6.5 & 17.2 & 2.1 & 0.2 & -8.7 & -3.6 & -0.2 & -4.2 & -3.5 & -8.9 & -3.6 & 3.9 & -1.5 & 0.6 & 1.0 & 1.07 \\
\hline $0.22 \div 0.34$ & 163 & 12.7 & 4.5 & 11.9 & 2.0 & 1.1 & -5.9 & -2.0 & 0.5 & -1.5 & -3.2 & -4.1 & -2.1 & 1.4 & -0.9 & 0.6 & $1.02 \pm 0.04$ & 1.05 \\
\hline $0.34 \div 0.46$ & 144 & 11.2 & 4.3 & 10.4 & 1.6 & 2.8 & -4.2 & -0.4 & 0.8 & -0.1 & -3.1 & -2.3 & -1.3 & 1.0 & -1.1 & 0.3 & $1.06 \pm 0.04$ & 1.04 \\
\hline $0.46 \div 0.58$ & 106 & 11.2 & 5.0 & 10.0 & 1.2 & 3.2 & -3.2 & 0.7 & 0.8 & 0.9 & -3.1 & -1.0 & -0.6 & 1.9 & -0.3 & 0.4 & $1.13 \pm 0.03$ & 1.02 \\
\hline $0.58 \div 0.70$ & 76.5 & 12.4 & 7.0 & 10.2 & 0.7 & 4.3 & -2.3 & 1.0 & 0.6 & 1.6 & -3.3 & 0.3 & 0.4 & 1.2 & -1.5 & 0.2 & $1.17 \pm 0.02$ & 0.97 \\
\hline$x_{\mathbb{P}}$ & $\begin{array}{c}d \sigma / d x_{\mathbb{P}} \\
{[\mathrm{pb}]}\end{array}$ & $\begin{array}{l}\delta_{\text {tot }} \\
{[\%]}\end{array}$ & $\begin{array}{c}\delta_{\text {stat }} \\
{[\%]}\end{array}$ & $\begin{array}{l}\delta_{\text {sys }} \\
{[\%]}\end{array}$ & $\begin{array}{c}\delta_{\theta} \\
{[\%]}\end{array}$ & $\begin{array}{c}\delta_{E} \\
{[\%]}\end{array}$ & $\begin{array}{c}\delta_{\mathrm{HFS}} \\
{[\%]} \\
\end{array}$ & $\begin{array}{l}\delta_{Q^{2}} \\
{[\%]}\end{array}$ & $\begin{array}{l}\delta_{x_{\mathbb{P}}} \\
{[\%]} \\
\end{array}$ & $\begin{array}{c}\delta_{\beta} \\
{[\%]} \\
\end{array}$ & $\begin{array}{c}\delta_{p *_{T, 1}} \\
{[\%]} \\
\end{array}$ & $\begin{array}{l}\delta_{z_{\mathbb{P}}} \\
{[\%]}\end{array}$ & $\begin{array}{c}\delta_{x_{\text {dijet }}} \\
{[\%]}\end{array}$ & $\begin{array}{c}\delta_{\Delta \eta *} \\
{[\%]} \\
\end{array}$ & $\begin{array}{c}\delta_{t} \\
{[\%]}\end{array}$ & $\begin{array}{l}\delta_{\mathrm{bgr}} \\
{[\%]}\end{array}$ & $1+\delta_{\text {had }}$ & $1+\delta_{\text {rad }}$ \\
\hline$-2.30 \div-2.10$ & 14.2 & 42.0 & 36.2 & 21.1 & 1.8 & 3.9 & -9.3 & -2.7 & 4.0 & -3.7 & -5.8 & -11.4 & -4.7 & 7.6 & -1.2 & 0.7 & $1.17 \pm 0.13$ & 1.06 \\
\hline$-2.10 \div-1.90$ & 53.5 & 14.7 & 8.9 & 11.7 & 1.6 & 2.4 & -5.6 & -0.6 & 1.2 & -0.8 & -3.2 & -3.7 & -1.8 & 2.3 & -1.4 & 0.0 & $1.10 \pm 0.08$ & 1.04 \\
\hline$-1.90 \div-1.70$ & 111 & 11.6 & 5.5 & 10.2 & 1.5 & 1.3 & -4.5 & -1.1 & 0.1 & -0.2 & -3.5 & -1.5 & -1.0 & 1.4 & -1.1 & 0.0 & $1.06 \pm 0.04$ & 1.04 \\
\hline$-1.70 \div-1.52$ & 196 & 10.9 & 4.9 & 9.8 & 1.3 & 2.5 & -3.6 & -1.0 & -0.5 & 0.5 & -3.4 & -0.0 & -0.3 & -0.5 & -0.5 & 0.8 & $1.03 \pm 0.03$ & 1.03 \\
\hline$z_{\mathbb{P}}$ & $\begin{array}{c}d \sigma / d z_{\mathbb{P}} \\
{[\mathrm{pb}]}\end{array}$ & $\begin{array}{l}\delta_{\text {tot }} \\
{[\%]}\end{array}$ & $\begin{array}{c}\delta_{\text {stat }} \\
{[\%]}\end{array}$ & $\begin{array}{l}\delta_{\text {sys }} \\
{[\%]}\end{array}$ & $\begin{array}{c}\delta_{\theta} \\
{[\%]}\end{array}$ & $\begin{array}{c}\delta_{E} \\
{[\%]}\end{array}$ & $\begin{array}{c}\delta_{\mathrm{HFS}} \\
{[\%]}\end{array}$ & $\begin{array}{l}\delta_{Q^{2}} \\
{[\%]} \\
\end{array}$ & $\begin{array}{l}\delta_{x_{P}} \\
{[\%]} \\
\end{array}$ & $\begin{array}{c}\delta_{\beta} \\
{[\%]} \\
\end{array}$ & $\begin{array}{c}\delta_{p *_{T, 1}} \\
{[\%]} \\
\end{array}$ & $\begin{array}{l}\delta_{z_{\mathbb{P}}} \\
{[\%]}\end{array}$ & $\begin{array}{c}\delta_{x_{\text {dijet }}} \\
{[\%]} \\
\end{array}$ & $\begin{array}{c}\delta_{\Delta \eta *} \\
{[\%]} \\
\end{array}$ & $\begin{array}{c}\delta_{t} \\
{[\%]}\end{array}$ & $\begin{array}{l}\delta_{\mathrm{bgr}} \\
{[\%]}\end{array}$ & $1+\delta_{\text {had }}$ & $1+\delta_{\mathrm{rad}}$ \\
\hline $0.00 \div 0.22$ & 70.4 & 20.3 & 9.3 & 18.0 & 1.4 & 3.4 & -4.0 & 1.2 & -0.5 & 4.6 & -2.4 & 12.0 & 4.1 & 6.5 & -0.5 & 0.8 & $1.10 \pm 0.03$ & 1.06 \\
\hline $0.22 \div 0.40$ & 132 & 11.9 & 6.3 & 10.1 & 1.5 & 3.0 & -1.2 & -0.9 & 0.3 & -0.1 & -3.9 & -2.3 & -2.2 & 1.0 & -0.9 & 0.4 & $1.07 \pm 0.02$ & 1.04 \\
\hline $0.40 \div 0.60$ & 89.7 & 14.9 & 6.8 & 13.3 & 1.2 & 1.6 & -9.1 & -1.3 & 0.8 & -1.2 & -2.8 & -3.9 & -1.4 & 0.5 & -0.6 & 0.3 & $1.10 \pm 0.03$ & 1.02 \\
\hline $0.60 \div 0.80$ & 54.8 & 14.9 & 7.5 & 12.9 & 2.5 & 1.9 & 7.6 & -1.4 & 0.9 & -1.2 & -3.2 & -4.2 & -1.4 & 0.2 & -2.0 & 0.1 & $1.10 \pm 0.10$ & 1.02 \\
\hline $0.80 \div 1.00$ & 19.9 & 45.0 & 11.4 & 43.5 & 0.8 & 0.6 & -42.1 & -1.9 & 1.3 & -2.4 & -2.5 & -5.1 & -2.0 & 3.0 & -1.5 & 0.6 & $0.57 \pm 0.10$ & 1.00 \\
\hline
\end{tabular}

Table 2: Diffractive DIS dijet cross section measured differentially as a function of $Q^{2}, y, \log x_{\mathbb{P}}$ and $z_{\mathbb{P}}$. The statistical $\delta_{\text {stat }}$ and systematic $\delta_{\text {sys }}$ uncertainties are given together with the total uncertainty $\delta_{\text {tot }}$. The next 12 columns represent $+1 \sigma$ shifts for the systematic error contributions from: electron polar angle measurement $\delta_{\theta}$, electron energy scale $\delta_{E}$, HFS energy scale $\delta_{\mathrm{HFS}}$, model uncertainties $\delta_{Q^{2}}, \delta_{x_{\mathbb{P}}}, \delta_{\beta}, \delta_{p *_{T, 1}}, \delta_{z_{\mathbb{P}}}, \delta_{x_{\mathrm{dijet}}}, \delta_{\Delta \eta *}$ and $\delta_{t}$ and the background normalisation uncertainty $\delta_{\mathrm{bgr}}$. The global normalisation uncertainty of $7.8 \%$ is not listed explicitly but is included in the total systematic uncertainty $\delta_{\text {sys }}$. The last two column show the correction factors for hadronisation and QED radiation, respectively.

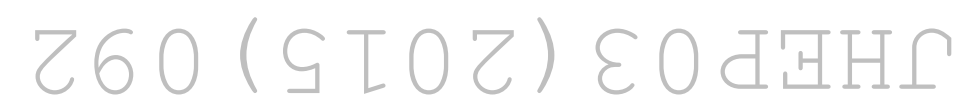




\begin{tabular}{|c|c|c|c|c|c|c|c|c|c|c|c|c|c|c|c|c|c|c|}
\hline $\begin{array}{c}p_{T, 1}^{*} \\
{[\mathrm{GeV}]}\end{array}$ & $\begin{array}{l}/ d p_{T, 1}^{*} \\
\mathrm{~s} / \mathrm{GeV}]\end{array}$ & $\begin{array}{l}\delta_{\text {tot }} \\
{[\%]}\end{array}$ & $\begin{array}{l}\delta_{\text {stat }} \\
{[\%]}\end{array}$ & $\begin{array}{l}\text { ys } \\
\text { y] }\end{array}$ & $\begin{array}{c}\delta_{\theta} \\
{[\%]}\end{array}$ & $\begin{array}{l}\delta_{E} \\
{[\%]}\end{array}$ & $\begin{array}{c}\delta_{\mathrm{HFS}} \\
{[\%]}\end{array}$ & $\begin{array}{l}\delta_{Q^{2}} \\
{[\%]}\end{array}$ & $\delta_{x_{P}}$ & $\begin{array}{c}\delta_{\beta} \\
{[\%]}\end{array}$ & $\begin{array}{c}\delta_{p *_{T}, 1} \\
{[\%]}\end{array}$ & $\begin{array}{l}\delta_{z_{\mathbb{P}}} \\
{[\%]}\end{array}$ & $\begin{array}{c}\delta_{x_{\text {dijet }}} \\
{[\%]}\end{array}$ & $\begin{array}{c}\delta_{\Delta \eta *} \\
{[\%]}\end{array}$ & $\begin{array}{c}\delta_{t} \\
{[\%]}\end{array}$ & $\begin{array}{l}\delta_{\text {bgr }} \\
{[\%]}\end{array}$ & $1+\delta_{\text {had }}$ & $1+\delta$ \\
\hline $5.50 \div 7.00$ & 30.8 & .6 & & & & 5 & -3.1 & -0.9 & .5 & & -1.3 & 1.7 & 21 & 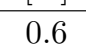 & & 1 & & 03 \\
\hline$\div s$ & & & & & & & 4.6 & & & & & & & & & & & \\
\hline$\div 15$ & 07 & & & & & & 9.8 & & & & -4 . & & -1 & & & & & 1.00 \\
\hline $\begin{array}{c}p_{T, 2}^{*} \\
{[G e V]}\end{array}$ & & [\%] & & $\begin{array}{l}\text { sys } \\
\%]\end{array}$ & $\begin{array}{c}\delta_{\theta} \\
{[\%]}\end{array}$ & 0] & $\begin{array}{c}\delta_{\mathrm{HFS}} \\
{[\%]}\end{array}$ & 0] & & $\begin{array}{c}\delta_{\beta} \\
{[\%]}\end{array}$ & $\begin{array}{c}\delta_{p *_{T, 1}} \\
{[\%]}\end{array}$ & 70] & {$[\%]$} & & $\begin{array}{c}\delta_{t} \\
{[\%]}\end{array}$ & & & \\
\hline $4.00 \div 6.5$ & 2.3 & & 3.7 & & & 2.3 & 3.8 & 1.0 & 0. & 08 & -1.2 & 2.0 & -1.2 & 1.2 & -0.8 & 1 & & 03 \\
\hline $0 \div$ & 5.67 & & & & & & -4.9 & -0.6 & & -0. & -2 & .3 & -0 & & -1 & & & \\
\hline $00 \div 15.0$ & 39 & & $2 .: 3$ & & 1.1 & 1.5 & -7 & 0. & 0. & $0.8>>$ & -6.2 & -2.4 & -0. & 2.8 & -1 & 1 & $0.97=$ & 1.06 \\
\hline $\begin{array}{c}\left\langle p_{T}^{*}\right\rangle \\
{[\mathrm{GeV}]}\end{array}$ & & & & & $\begin{array}{c}\delta_{\theta} \\
{[\%]}\end{array}$ & $\delta_{E}$ & $\begin{array}{c}\delta_{\mathrm{HFS}} \\
{[\%]}\end{array}$ & $\begin{array}{l}\delta_{Q^{2}} \\
{[\%]}\end{array}$ & & $\delta$ & $\begin{array}{c}\delta_{p_{* T, 1}} \\
{[\%]}\end{array}$ & $\begin{array}{l}\delta_{z_{P}} \\
{[\%]}\end{array}$ & $\begin{array}{c}\delta_{x_{\text {dijet }}} \\
{[\%]}\end{array}$ & & $\begin{array}{c}\delta_{t} \\
{[\%]}\end{array}$ & & inc & \\
\hline $4.75 \div 6.5$ & $e^{2} \rightarrow$ & 9. & 3 & & & 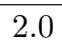 & -3.3 & 1.1 & 0 . & & -1. & 1.9 & -1 & 0 . & -0.8 & 1 & & \\
\hline $6.50 \div 9.0$ & 8.52 & & & & & 2.4 & .0 & 4 & 0.8 & -0.1 & -1.7 & 8 & -0 & & -1.1 & & & 1.04 \\
\hline $00 \div 15.00$ & 701 & 9.7 & 3.4 & 4.4 & 0.7 & 1.2 & -9.2 & -0.3 & 0.7 & -0.2 & -5.4 & -3.5 & -1.3 & 3.8 & -0.9 & 0.5 & $=0.03$ & 1.06 \\
\hline$\Delta \eta$ & $\begin{array}{c}\sigma / d \Delta \eta^{*} \\
{[\mathrm{pb}]}\end{array}$ & $\begin{array}{l}\delta_{\text {tot }} \\
{[\%]}\end{array}$ & & $\begin{array}{l}\delta_{\text {sys }} \\
{[\%]}\end{array}$ & $\begin{array}{c}\delta_{\theta} \\
{[\%]}\end{array}$ & $\begin{array}{l}\delta_{E} \\
{[\%]}\end{array}$ & $\begin{array}{c}\delta_{\mathrm{HFS}} \\
{[\%]}\end{array}$ & $\delta_{Q^{2}}$ & $\begin{array}{l}\delta_{x_{P}} \\
{[\%]}\end{array}$ & $\delta_{\beta}$ & $\begin{array}{c}\delta_{p *_{T, 1}} \\
{[\%]}\end{array}$ & $\begin{array}{l}\delta_{z_{\mathbb{P}}} \\
{[\%]}\end{array}$ & $\begin{array}{c}\delta_{x_{\text {dijet }}} \\
{[\%]}\end{array}$ & & $\begin{array}{c}\delta_{t} \\
{[\%]}\end{array}$ & & $\delta_{\mathrm{ha}}$ & $+\delta_{1}$ \\
\hline 0. & 51.6 & & 9 & & & 2. & -4.4 & -1.0 & 1. & & $-3.8>$ & 2.3 & -1.4 & 10.6 & -1.2 & & & \\
\hline 0. & 57.8 & & 7 & & & & .1 & & 0. & & -3.0 & 1 & 5 & 6. & 1.1 & & & .04 \\
\hline $0.40 \div 0.80$ & 5.1 & & 5. & & 1.9 & 2.5 & -4 & & 0. & & -3.8 & & 2 & & 3 & & & 04 \\
\hline & & & & & & 2 & -4 & -1 & 0. & & -3 & & -1.0 & - & & & & .03 \\
\hline $0 \div 3.00$ & 9.29 & 5.0 & 6.7 & 3.4 & 1.2 & 3.4 & -5.3 & -1.0 & 0.2 & -0.0 & -2.8 & -3.4 & -1.2 & -7.4 & -1.1 & 0.3 & $1.04 \pm 0.06$ & 1.03 \\
\hline
\end{tabular}

Table 3: Diffractive DIS dijet cross section measured differentially as a function of $p_{T, 1}^{*}, p_{T, 2}^{*},\left\langle p_{T}^{*}\right\rangle$ and $\Delta \eta^{*}$. The statistical $\delta_{\text {stat }}$ and systematic $\delta_{\text {sys }}$ uncertainties are given together with the total uncertainty $\delta_{\text {tot }}$. Further details are given in table 2 . 


\begin{tabular}{|c|c|c|c|c|c|c|c|c|c|c|c|c|c|c|c|c|c|c|c|c|}
\hline & $z_{\mathbb{P}}$ & $\begin{array}{c}Q^{2} \\
{\left[\mathrm{GeV}^{2}\right]}\end{array}$ & $\begin{array}{c}\frac{d^{2} \sigma}{d z_{z_{P}} d Q^{2}} \\
{\left[\mathrm{pb} / \mathrm{GeV}^{2}\right]}\end{array}$ & $\begin{array}{l}\delta_{\text {tot }} \\
{[\%]}\end{array}$ & $\begin{array}{c}\delta_{\text {stat }} \\
{[\%]}\end{array}$ & $\begin{array}{l}\delta_{\mathrm{sys}} \\
{[\%]} \\
\end{array}$ & $\begin{array}{c}\delta_{\theta} \\
{[\%]}\end{array}$ & $\begin{array}{c}\delta_{E} \\
{[\%]}\end{array}$ & $\begin{array}{c}\delta_{\mathrm{HFS}} \\
{[\%]}\end{array}$ & $\begin{array}{l}\delta_{Q^{2}} \\
{[\%]}\end{array}$ & $\begin{array}{l}\delta_{x_{\mathbb{P}}} \\
{[\%]}\end{array}$ & $\begin{array}{c}\delta_{\beta} \\
{[\%]} \\
\end{array}$ & $\begin{array}{c}\delta_{p *_{T, 1}} \\
{[\%]}\end{array}$ & $\begin{array}{l}\delta_{z_{\mathbb{P}}} \\
{[\%]}\end{array}$ & $\begin{array}{c}\delta_{x_{\text {dijet }}} \\
{[\%]}\end{array}$ & $\begin{array}{c}\delta_{\Delta \eta *} \\
{[\%]}\end{array}$ & $\begin{array}{c}\delta_{t} \\
{[\%]}\end{array}$ & $\begin{array}{l}\delta_{\mathrm{bgr}} \\
{[\%]}\end{array}$ & $1+\delta_{\text {had }}$ & $1+\delta_{\mathrm{rad}}$ \\
\hline & $0.0 \div 0.3$ & $4 \div 10$ & 7.67 & 14.8 & 7.7 & 12.7 & 2.0 & 3.5 & -1.0 & 1.6 & -0.3 & 3.4 & -3.3 & 6.5 & 2.2 & 3.1 & -1.3 & 0.5 & $1.08 \pm 0.03$ & 1.05 \\
\hline & & $10 \div 20$ & 2.40 & 15.6 & 10.0 & 12.1 & 0.7 & 2.9 & -2.1 & -2.0 & -0.2 & 1.1 & -3.2 & 5.7 & 1.5 & 4.6 & -0.4 & 0.7 & $1.08 \pm 0.02$ & 1.05 \\
\hline & & $20 \div 40$ & 0.544 & 27.6 & 20.8 & 18.2 & 2.2 & 4.7 & -3.3 & 0.9 & -0.2 & 4.7 & -3.5 & 11.6 & 2.8 & 7.3 & -0.4 & 0.2 & $1.09 \pm 0.02$ & 1.05 \\
\hline & & $40 \div 100$ & 0.0994 & 41.6 & 35.7 & 21.3 & 1.2 & 7.3 & -3.3 & -0.4 & -0.1 & 4.1 & -4.1 & 12.9 & 3.1 & 10.0 & -3.7 & 2.0 & $1.09 \pm 0.02$ & 1.06 \\
\hline & $0.3 \div 0.5$ & $4 \div 10$ & 8.80 & 18.4 & 9.3 & 15.9 & 2.0 & 3.1 & -8.6 & -0.7 & 1.2 & -1.0 & -5.3 & -7.4 & -3.9 & -0.6 & -1.5 & 0.1 & $1.08 \pm 0.02$ & 1.03 \\
\hline & & $10 \div 20$ & 2.31 & 19.9 & 13.6 & 14.5 & 2.0 & 2.4 & -6.9 & -0.4 & 0.7 & -1.5 & -3.3 & -7.7 & -4.1 & -0.0 & -0.4 & 1.0 & $1.08 \pm 0.02$ & 1.03 \\
\hline & & $20 \div 40$ & 1.12 & 17.0 & 12.6 & 11.4 & -0.4 & 3.2 & -3.7 & 0.2 & 0.5 & -0.7 & -3.0 & -5.3 & -2.8 & -0.3 & -0.0 & 0.2 & $1.08 \pm 0.03$ & 1.02 \\
\hline 1 & & $40 \div 100$ & 0.264 & 20.1 & 17.1 & 10.6 & 0.7 & 2.6 & -4.4 & -0.2 & 0.4 & -0.9 & -2.1 & -3.4 & -2.1 & 1.4 & 1.3 & 0.2 & $1.07 \pm 0.03$ & 1.03 \\
\hline$\bullet$ & $0.5 \div 0.7$ & $4 \div 10$ & 4.50 & 17.8 & 13.3 & 11.8 & 3.3 & 1.9 & 6.5 & -1.4 & 1.1 & -0.7 & -3.1 & -2.8 & -0.4 & 0.9 & -0.7 & 0.2 & $1.14 \pm 0.06$ & 1.03 \\
\hline 1 & & $10 \div 20$ & 1.86 & 15.2 & 11.8 & 9.6 & 0.8 & 1.0 & 3.1 & -0.5 & 0.5 & -0.4 & -3.1 & -2.6 & -0.6 & 0.2 & -1.8 & 0.1 & $1.12 \pm 0.06$ & 1.02 \\
\hline & & $20 \div 40$ & 0.703 & 16.2 & 13.5 & 8.9 & 2.0 & 0.8 & -0.2 & -0.7 & 0.5 & -0.6 & -2.2 & -2.3 & -0.7 & 0.2 & -1.6 & 0.2 & $1.12 \pm 0.06$ & 1.02 \\
\hline & & $40 \div 100$ & 0.109 & 31.9 & 29.7 & 11.4 & 2.2 & -0.8 & 3.2 & -0.8 & 0.1 & -1.3 & -1.4 & -4.0 & -1.0 & -1.6 & -5.6 & 0.1 & $1.12 \pm 0.06$ & 1.01 \\
\hline & $0.7 \div 1.0$ & $4 \div 10$ & 1.99 & 27.8 & 11.7 & 25.2 & 2.2 & 2.9 & -21.9 & -1.6 & 1.8 & -1.9 & -3.8 & -6.5 & -2.6 & 1.7 & -2.9 & 0.3 & $0.79 \pm 0.11$ & 1.02 \\
\hline & & $10 \div 20$ & 0.639 & 26.9 & 11.2 & 24.5 & 1.4 & 0.4 & -22.1 & -0.4 & 1.1 & -1.6 & -1.7 & -5.2 & -2.0 & 2.4 & -1.8 & 0.3 & $0.81 \pm 0.11$ & 1.01 \\
\hline & & $20 \div 40$ & 0.248 & 22.4 & 13.0 & 18.2 & 0.9 & 1.6 & -15.3 & -0.3 & 0.9 & -1.1 & -2.6 & -4.3 & -1.6 & 1.1 & -0.3 & 0.0 & $0.85 \pm 0.11$ & 1.00 \\
\hline & & $40 \div 100$ & 0.0968 & 18.5 & 13.3 & 13.0 & 0.3 & 2.1 & -9.0 & -0.5 & 0.4 & -1.1 & -2.2 & -3.4 & -1.5 & 1.3 & 0.4 & 0.5 & $0.89 \pm 0.10$ & 1.01 \\
\hline
\end{tabular}

Table 4: Diffractive DIS dijet cross section measured differentially as a function of $z_{\mathbb{P}}$ and $Q^{2}$. The statistical $\delta_{\text {stat }}$ and systematic $\delta_{\text {sys }}$ uncertainties are given together with the total uncertainty $\delta_{\text {tot }}$. Further details are given in table 2 . 


\begin{tabular}{|c|c|c|c|c|c|c|c|c|c|c|c|c|c|c|c|c|c|c|c|c|}
\hline & $\begin{array}{c}p_{\mathrm{T}, 1}^{*} \\
{\left[\mathrm{GeV}^{2}\right]}\end{array}$ & $\begin{array}{c}Q^{2} \\
{[\mathrm{GeV}]}\end{array}$ & $\begin{array}{c}\frac{d^{2} \sigma}{d p_{\mathrm{T}, 1}^{*} d Q^{2}} \\
{\left[\mathrm{pb} / \mathrm{GeV}^{3}\right]}\end{array}$ & $\begin{array}{l}\delta_{\text {tot }} \\
{[\%]}\end{array}$ & $\begin{array}{l}\delta_{\text {stat }} \\
{[\%]}\end{array}$ & $\begin{array}{l}\delta_{\text {sys }} \\
{[\%]}\end{array}$ & $\begin{array}{c}\delta_{\theta} \\
{[\%]}\end{array}$ & $\begin{array}{l}\delta_{E} \\
{[\%]}\end{array}$ & $\begin{array}{c}\delta_{\mathrm{HFS}} \\
{[\%]}\end{array}$ & $\begin{array}{l}\delta_{Q^{2}} \\
{[\%]}\end{array}$ & $\begin{array}{l}\delta_{x_{\mathbb{P}}} \\
{[\%]}\end{array}$ & $\begin{array}{c}\delta_{\beta} \\
{[\%]}\end{array}$ & $\begin{array}{c}\delta_{p *_{T, 1}} \\
{[\%]}\end{array}$ & $\begin{array}{l}\delta_{z_{\mathbb{P}}} \\
{[\%]}\end{array}$ & $\begin{array}{c}\delta_{x_{\mathrm{dijet}}} \\
{[\%]}\end{array}$ & $\begin{array}{c}\delta_{\Delta \eta *} \\
{[\%]}\end{array}$ & $\begin{array}{c}\delta_{t} \\
{[\%]}\end{array}$ & $\begin{array}{l}\delta_{\mathrm{bgr}} \\
{[\%]}\end{array}$ & $1+\delta_{\text {had }}$ & $1+\delta_{\mathrm{rad}}$ \\
\hline & $5.5 \div 7.0$ & $4 \div 6$ & 3.35 & 15.6 & 9.1 & 12.7 & 0.4 & 5.9 & -1.2 & 2.7 & 1.1 & 3.4 & -1.3 & 5.8 & 2.9 & 0.6 & -1.0 & 0.1 & $1.05 \pm 0.05$ & 1.04 \\
\hline & & $6 \div 10$ & 1.84 & 12.7 & 7.1 & 10.5 & 3.0 & 0.8 & -4.7 & -1.5 & 0.3 & -1.0 & -1.8 & -2.9 & -1.3 & 0.7 & -1.6 & 0.1 & $1.05 \pm 0.05$ & 1.02 \\
\hline & & $10 \div 18$ & 0.834 & 12.3 & 7.2 & 9.9 & 1.2 & 0.9 & -3.2 & -1.1 & 0.5 & -1.4 & -1.6 & -3.9 & -1.7 & 0.6 & -1.1 & 0.2 & $1.05 \pm 0.05$ & 1.02 \\
\hline & & $18 \div 34$ & 0.344 & 13.3 & 8.6 & 10.1 & 1.5 & 0.4 & -5.1 & 0.3 & 0.5 & -0.4 & -1.4 & -2.6 & -1.7 & 1.1 & -0.0 & 0.0 & $1.06 \pm 0.05$ & 1.03 \\
\hline & & $34 \div 100$ & 0.0613 & 15.8 & 11.7 & 10.6 & 1.5 & 0.7 & -5.0 & -0.4 & 0.5 & -1.1 & -1.7 & -3.4 & -2.0 & 0.9 & -1.6 & 0.1 & $1.07 \pm 0.04$ & 1.02 \\
\hline & $7.0 \div 9.0$ & $4 \div 6$ & 1.23 & 18.6 & 15.1 & 10.9 & -0.1 & 3.0 & -6.1 & 1.3 & 1.2 & 1.5 & -2.5 & 0.1 & -0.7 & 0.0 & -0.3 & 0.5 & $1.06 \pm 0.04$ & 1.05 \\
\hline & & $6 \div 10$ & 0.578 & 16.4 & 12.9 & 10.1 & 1.9 & 3.3 & -3.9 & 1.1 & 0.8 & 0.0 & -0.6 & -2.1 & -1.3 & 1.5 & -0.8 & 0.4 & $1.06 \pm 0.04$ & 1.05 \\
\hline & & $10 \div 18$ & 0.287 & 16.6 & 12.6 & 10.7 & 0.4 & 3.1 & -6.0 & -0.3 & 0.7 & -0.1 & -1.3 & -0.3 & -0.4 & 2.3 & 0.7 & 0.6 & $1.06 \pm 0.05$ & 1.04 \\
\hline & & $18 \div 34$ & 0.100 & 20.4 & 17.6 & 10.3 & 0.3 & 5.2 & -3.5 & -0.2 & 0.8 & -0.2 & -0.5 & -1.0 & -0.4 & 0.0 & -1.5 & 0.7 & $1.07 \pm 0.04$ & 1.04 \\
\hline & & $34 \div 100$ & 0.0276 & 19.9 & 17.4 & 9.6 & -0.6 & 3.7 & -3.1 & -0.6 & 0.5 & -1.0 & -0.6 & -1.6 & -0.4 & 1.0 & 1.5 & 0.6 & $1.06 \pm 0.06$ & 1.04 \\
\hline & $9.0 \div 15.0$ & $4 \div 6$ & 0.122 & 30.1 & 26.6 & 14.2 & 7.8 & 0.5 & -5.5 & -0.1 & 0.7 & 0.3 & -5.5 & -1.2 & -0.5 & 3.3 & -2.2 & 0.8 & $1.04 \pm 0.03$ & 1.06 \\
\hline & & $6 \div 10$ & 0.0511 & 30.4 & 24.7 & 17.8 & 1.9 & 1.1 & -12.4 & -0.6 & 1.3 & -0.0 & -6.3 & -2.8 & -0.9 & 6.1 & -3.0 & 0.4 & $1.03 \pm 0.03$ & 1.05 \\
\hline & & $10 \div 18$ & 0.0207 & 35.5 & 30.0 & 19.0 & 1.4 & 1.7 & -11.6 & -1.1 & 1.0 & -1.4 & -6.5 & -6.4 & -2.9 & 5.6 & -6.0 & 0.5 & $1.03 \pm 0.02$ & 1.05 \\
\hline & & $18 \div 34$ & 0.0160 & 24.5 & 20.1 & 14.0 & 1.6 & 1.8 & -8.2 & 0.0 & 0.6 & -0.1 & -4.6 & -2.6 & -0.6 & 5.2 & -2.5 & 0.4 & $1.04 \pm 0.04$ & 1.06 \\
\hline & & $34 \div 100$ & 0.0034 & 31.9 & 27.8 & 15.7 & 3.6 & 0.3 & -9.0 & 1.8 & 0.7 & 1.0 & -3.5 & -3.1 & -1.5 & 6.0 & -4.9 & 1.0 & $1.05 \pm 0.03$ & 1.07 \\
\hline
\end{tabular}

Table 5: Diffractive DIS dijet cross section measured differentially as a function of $p_{\mathrm{T}, 1}^{*}$ and $Q^{2}$. The statistical $\delta_{\text {stat }}$ and systematic $\delta_{\text {sys }}$ uncertainties are given together with the total uncertainty $\delta_{\text {tot. }}$. Further details are given in table 2 . 


\begin{tabular}{|c|c|ccccc|}
\hline$Q^{2}[\mathrm{GeV}]$ & \#Bin & 1 & 2 & 3 & 4 & 5 \\
\hline $4 \div 6$ & 1 & 100 & -5 & 5 & & \\
$6 \div 10$ & 2 & & 100 & 1 & 1 & \\
$10 \div 18$ & 3 & & & 100 & -2 & 1 \\
$18 \div 34$ & 4 & & & & 100 & 8 \\
$34 \div 100$ & 5 & & & & & 100 \\
\hline$y$ & $\#$ Bin & 1 & 2 & 3 & 4 & 5 \\
\hline $0.1 \div 0.2$ & 1 & 100 & -7 & 8 & 5 & 4 \\
$0.2 \div 0.3$ & 2 & & 100 & -6 & 8 & 4 \\
$0.3 \div 0.5$ & 3 & & & 100 & -4 & 7 \\
$0.5 \div 0.6$ & 4 & & & & 100 & -10 \\
$0.6 \div 0.7$ & 5 & & & & & 100 \\
\hline$x_{\mathbb{P}}$ & $\#$ Bin & 1 & 2 & 3 & 4 & \\
\hline$-2.30 \div-2.10$ & 1 & 100 & -55 & 17 & -2 & \\
$-2.10 \div-1.90$ & 2 & & 100 & -41 & 11 & \\
$-1.90 \div-1.70$ & 3 & & & 100 & -31 & \\
$-1.70 \div-1.52$ & 4 & & & & 100 & \\
\hline$z_{\mathbb{P}}$ & $\#$ Bin & 1 & 2 & 3 & 4 & 5 \\
\hline $0.0 \div 0.2$ & 1 & 100 & -24 & 8 & 1 & \\
$0.2 \div 0.4$ & 2 & & 100 & -31 & 10 & -2 \\
$0.4 \div 0.6$ & 3 & & & 100 & -45 & 17 \\
$0.6 \div 0.8$ & 4 & & & & 100 & -52 \\
$0.8 \div 1.0$ & 5 & & & & & 100 \\
\hline
\end{tabular}

Table 6: Correlation coefficients between data points for the single-differential measurements in $Q^{2}, y, x_{\mathbb{P}}$ and $z_{\mathbb{P}}$. The values are given in per cent. 


\begin{tabular}{|c|c|c|c|c|c|c|}
\hline$\overline{p_{\mathrm{T}, 1}^{*}[\mathrm{GeV}]}$ & $\# \operatorname{Bin}$ & 1 & 2 & 3 & & \\
\hline $5.5 \div 7.0$ & 1 & 100 & -26 & 1 & & \\
\hline $7.0 \div 9.0$ & 2 & & 100 & -54 & & \\
\hline $9.0 \div 15.0$ & 3 & & & 100 & & \\
\hline$p_{\mathrm{T}, 2}^{*}[\mathrm{GeV}]$ & $\# \operatorname{Bin}$ & 1 & 2 & 3 & & \\
\hline $4.0 \div 6.5$ & 1 & 100 & -36 & 13 & & \\
\hline $6.5 \div 9.0$ & 2 & & 100 & -46 & & \\
\hline $9.0 \div 15.0$ & 3 & & & 100 & & \\
\hline$\left\langle p_{\mathrm{T}}^{*}\right\rangle[\mathrm{GeV}]$ & $\# B i n$ & 1 & 2 & 3 & & \\
\hline $4.75 \div 6.50$ & 1 & 100 & -33 & 12 & & \\
\hline $6.50 \div 9.00$ & 2 & & 100 & -49 & & \\
\hline $9.00 \div 15.00$ & 3 & & & 100 & & \\
\hline$\Delta \eta^{*}$ & $\# \operatorname{Bin}$ & 1 & 2 & 3 & 4 & 5 \\
\hline $0.00 \div 0.15$ & 1 & 100 & -49 & 13 & 1 & 2 \\
\hline $0.15 \div 0.40$ & 2 & & 100 & -29 & 9 & 1 \\
\hline $0.40 \div 0.80$ & 3 & & & 100 & -19 & 7 \\
\hline $0.80 \div 1.30$ & 4 & & & & 100 & -20 \\
\hline $1.30 \div 3.00$ & 5 & & & & & 100 \\
\hline
\end{tabular}

Table 7: Correlation coefficients between data points for the single-differential measurements in $p_{\mathrm{T}, 1}^{*}, p_{\mathrm{T}, 2}^{*},\left\langle p_{\mathrm{T}}^{*}\right\rangle$ and $\Delta \eta^{*}$. The values are given in per cent.

\begin{tabular}{|c|c|c|c|c|c|c|c|c|c|c|c|c|c|c|c|c|c|c|}
\hline$z_{\mathbb{P}}$ & $Q^{2}\left[\mathrm{GeV}^{2}\right]$ & \#Bin & 1 & 2 & 3 & 4 & 5 & 6 & 7 & 8 & 9 & 10 & 11 & 12 & 13 & 14 & 15 & 16 \\
\hline \multirow[t]{4}{*}{$0.0 \div 0.3$} & $4 \div 10$ & 1 & 100 & 1 & & & -32 & 3 & & & 11 & & & & -3 & & & \\
\hline & $10 \div 20$ & 2 & & 100 & -2 & 1 & 3 & -41 & 2 & & & 19 & & & & -5 & & \\
\hline & $20 \div 40$ & 3 & & & 100 & 4 & & 2 & -37 & 4 & & & 20 & 1 & & & -4 & 1 \\
\hline & $40 \div 100$ & 4 & & & & 100 & & & 3 & -37 & & & 1 & 22 & & & 1 & -5 \\
\hline \multirow[t]{4}{*}{$0.3 \div 0.5$} & $4 \div 10$ & 5 & & & & & 100 & -3 & & & -46 & 2 & & & 15 & & & \\
\hline & $10 \div 20$ & 6 & & & & & & 100 & -3 & & 3 & -53 & 2 & & & 19 & & \\
\hline & $20 \div 40$ & 7 & & & & & & & 100 & -3 & & 2 & -51 & 2 & & -1 & 17 & \\
\hline & $40 \div 100$ & 8 & & & & & & & & 100 & & & 2 & -51 & & & & 21 \\
\hline \multirow[t]{4}{*}{$0.5 \div 0.7$} & $4 \div 10$ & 9 & & & & & & & & & 100 & -5 & & & -47 & 2 & & \\
\hline & $10 \div 20$ & 10 & & & & & & & & & & 100 & -3 & & 1 & -46 & 1 & \\
\hline & $20 \div 40$ & 11 & & & & & & & & & & & 100 & -2 & & 2 & -44 & 1 \\
\hline & $40 \div 100$ & 12 & & & & & & & & & & & & 100 & & & 1 & -51 \\
\hline \multirow[t]{4}{*}{$0.7 \div 1.0$} & $4 \div 10$ & 13 & & & & & & & & & & & & & 100 & -4 & & \\
\hline & $10 \div 20$ & 14 & & & & & & & & & & & & & & 100 & -5 & \\
\hline & $20 \div 40$ & 15 & & & & & & & & & & & & & & & 100 & -2 \\
\hline & $40 \div 100$ & 16 & & & & & & & & & & & & & & & & 100 \\
\hline
\end{tabular}

Table 8: Correlation coefficients between data points for the double-differential measurement in $z_{\mathbb{P}}$ and $Q^{2}$. The values are given in per cent. 


\begin{tabular}{|c|c|c|c|c|c|c|c|c|c|c|c|c|c|c|c|c|c|}
\hline$p_{\mathrm{T}, 1}^{*}[\mathrm{GeV}]$ & $Q^{2}\left[\mathrm{GeV}^{2}\right]$ & \#Bin & 1 & 2 & 3 & 4 & 5 & 6 & 7 & 8 & 9 & 10 & 11 & 12 & 13 & 14 & 15 \\
\hline \multirow[t]{5}{*}{$5.5 \div 7.0$} & $4 \div 6$ & 1 & 100 & -7 & 1 & & & -44 & 2 & & & & 13 & 1 & 1 & 1 & \\
\hline & $6 \div 10$ & 2 & & 100 & -3 & & & 3 & -57 & 3 & -1 & & & 17 & 1 & 1 & 1 \\
\hline & $10 \div 18$ & 3 & & & 100 & -2 & 1 & 1 & 3 & -59 & 1 & -1 & 2 & 1 & 22 & 1 & 1 \\
\hline & $18 \div 34$ & 4 & & & & 100 & 3 & -1 & & & -58 & 1 & 2 & 1 & 2 & 25 & 2 \\
\hline & $34 \div 100$ & 5 & & & & & 100 & & & & 1 & -56 & & & 1 & 2 & 27 \\
\hline \multirow[t]{5}{*}{$7.0 \div 9.0$} & $4 \div 6$ & 6 & & & & & & 100 & -7 & 3 & 3 & 1 & -60 & 2 & -5 & -6 & -3 \\
\hline & $6 \div 10$ & 7 & & & & & & & 100 & -4 & 2 & 1 & 3 & -57 & & -3 & -2 \\
\hline & $10 \div 18$ & 8 & & & & & & & & 100 & & 2 & -6 & & -60 & -4 & -4 \\
\hline & $18 \div 34$ & 9 & & & & & & & & & 100 & 1 & -7 & -3 & -6 & -62 & -3 \\
\hline & $34 \div 100$ & 10 & & & & & & & & & & 100 & -4 & -2 & -5 & -5 & -64 \\
\hline \multirow[t]{5}{*}{$9.0 \div 15.0$} & $4 \div 6$ & 11 & & & & & & & & & & & 100 & -5 & 13 & 14 & 7 \\
\hline & $6 \div 10$ & 12 & & & & & & & & & & & & 100 & & 6 & 3 \\
\hline & $10 \div 18$ & 13 & & & & & & & & & & & & & 100 & 10 & 9 \\
\hline & $18 \div 34$ & 14 & & & & & & & & & & & & & & 100 & 8 \\
\hline & $34 \div 100$ & 15 & & & & & & & & & & & & & & & 100 \\
\hline
\end{tabular}

Table 9: Correlation coefficients between data points for the double-differential measurement in $p_{\mathrm{T}, 1}^{*}$ and $Q^{2}$. The values are given in per cent. 

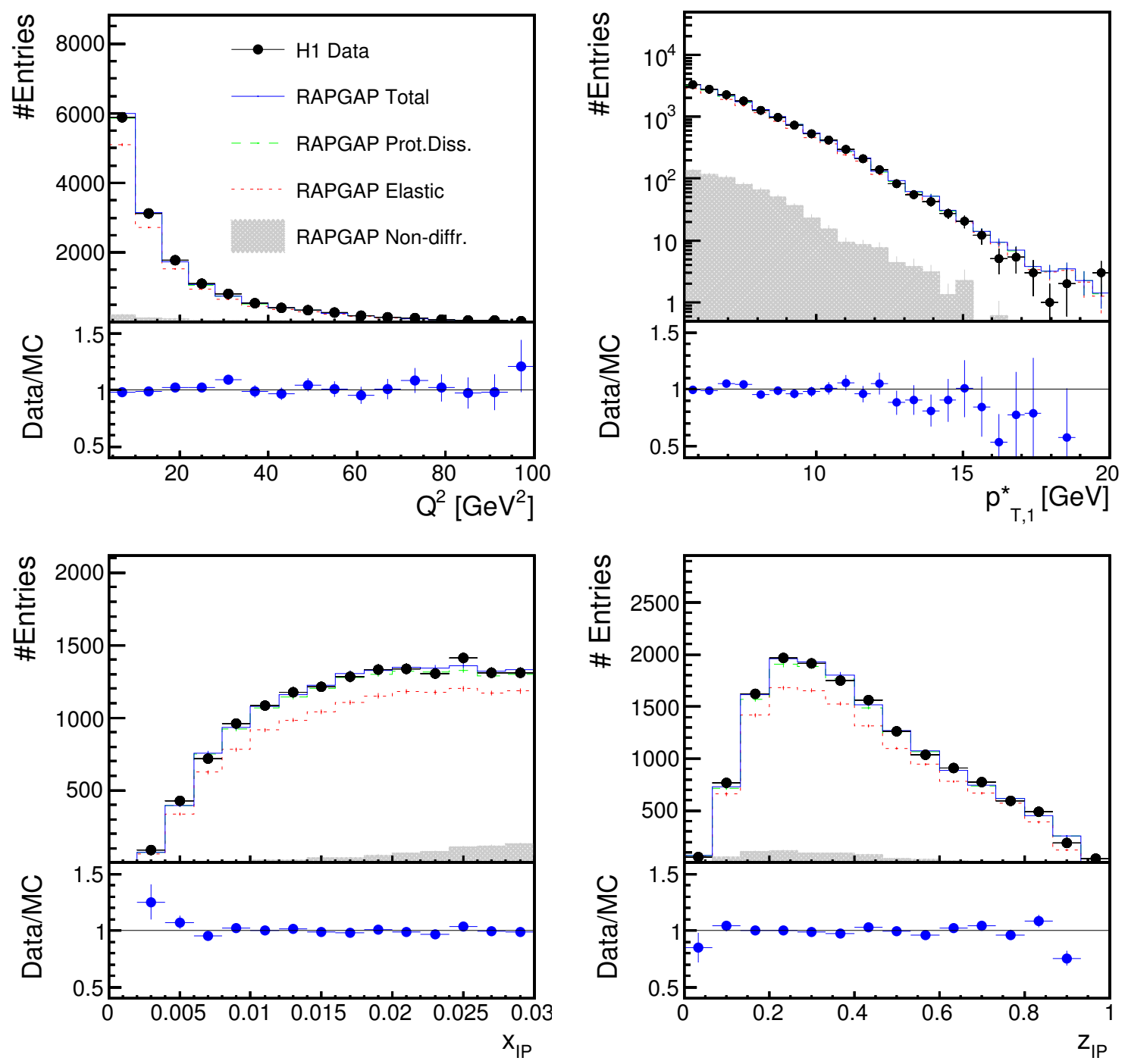

Figure 2: Distributions of the kinematic quantities $Q^{2}, p_{\mathrm{T}, 1}^{*}, x_{\mathbb{P}}$ and $z_{\mathbb{P}}$. The data are shown as black points compared to the sum of MC simulation estimates. The filled area shows the contribution of non-diffractive DIS, the dotted line shows the diffractive contribution with the elastically scattered proton added to the non-diffractive DIS and the dashed line displays the proton dissociation contribution added to the diffractive contribution with the elastically scattered proton and the non-diffractive DIS contribution. The sum of all contributions including the resolved photon processes is given by the full line. The MC is reweighted to the data. The ratio of data to the MC prediction is shown in the lower part of of the individual figures. 

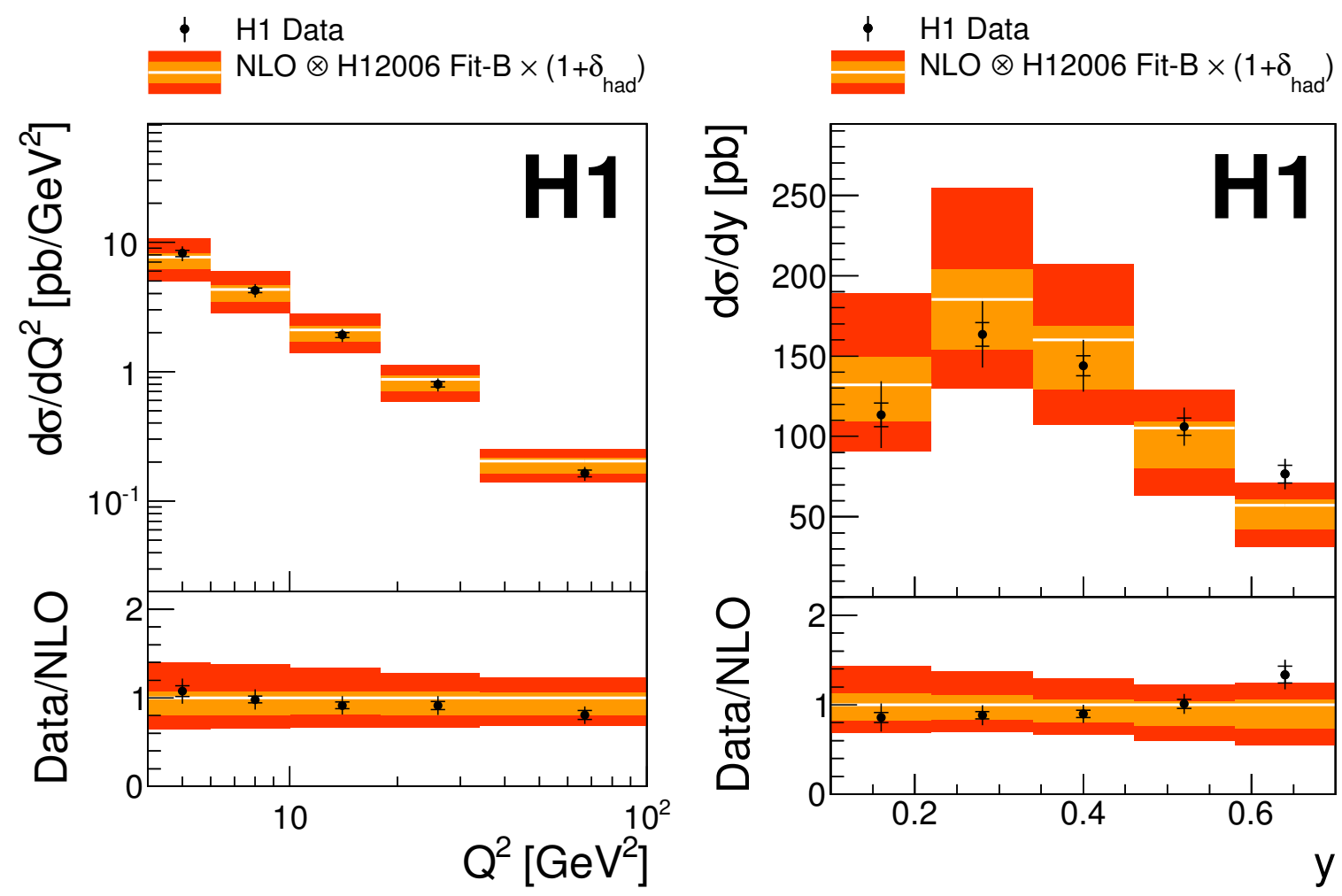

Figure 3: Diffractive dijet differential cross section as a function of $Q^{2}$ and $y$. The inner error bars on the data points represent the statistical uncertainties, while the outer error bars include the systematic uncertainties added in quadrature. The NLO QCD prediction based on the H12006 Fit-B DPDF set is displayed as a white line. The light shaded band indicates the uncertainty arising from hadronisation and the DPDF fit added in quadrature. The outer dark band shows the full theory uncertainty including the QCD scale uncertainty added in quadrature. The ratio of the single-differential cross section to the NLO prediction is shown in the lower part of the individual figures. 

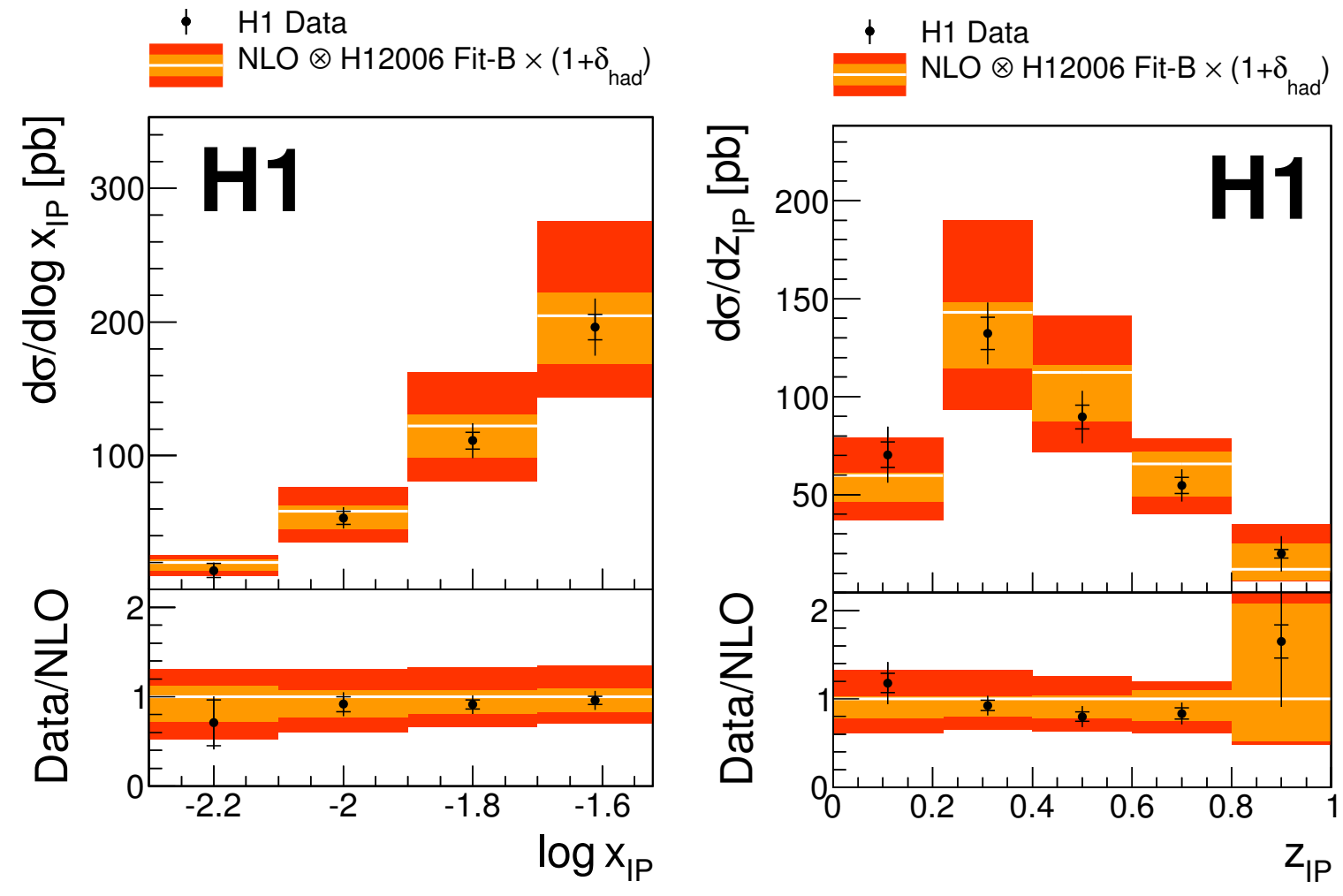

Figure 4: Diffractive dijet differential cross section as a function of $\log x_{\mathbb{P}}$ and $z_{\mathbb{P}}$. The inner error bars on the data points represent the statistical uncertainties, while the outer error bars include the systematic uncertainties added in quadrature. Further details are given in figure 3. 

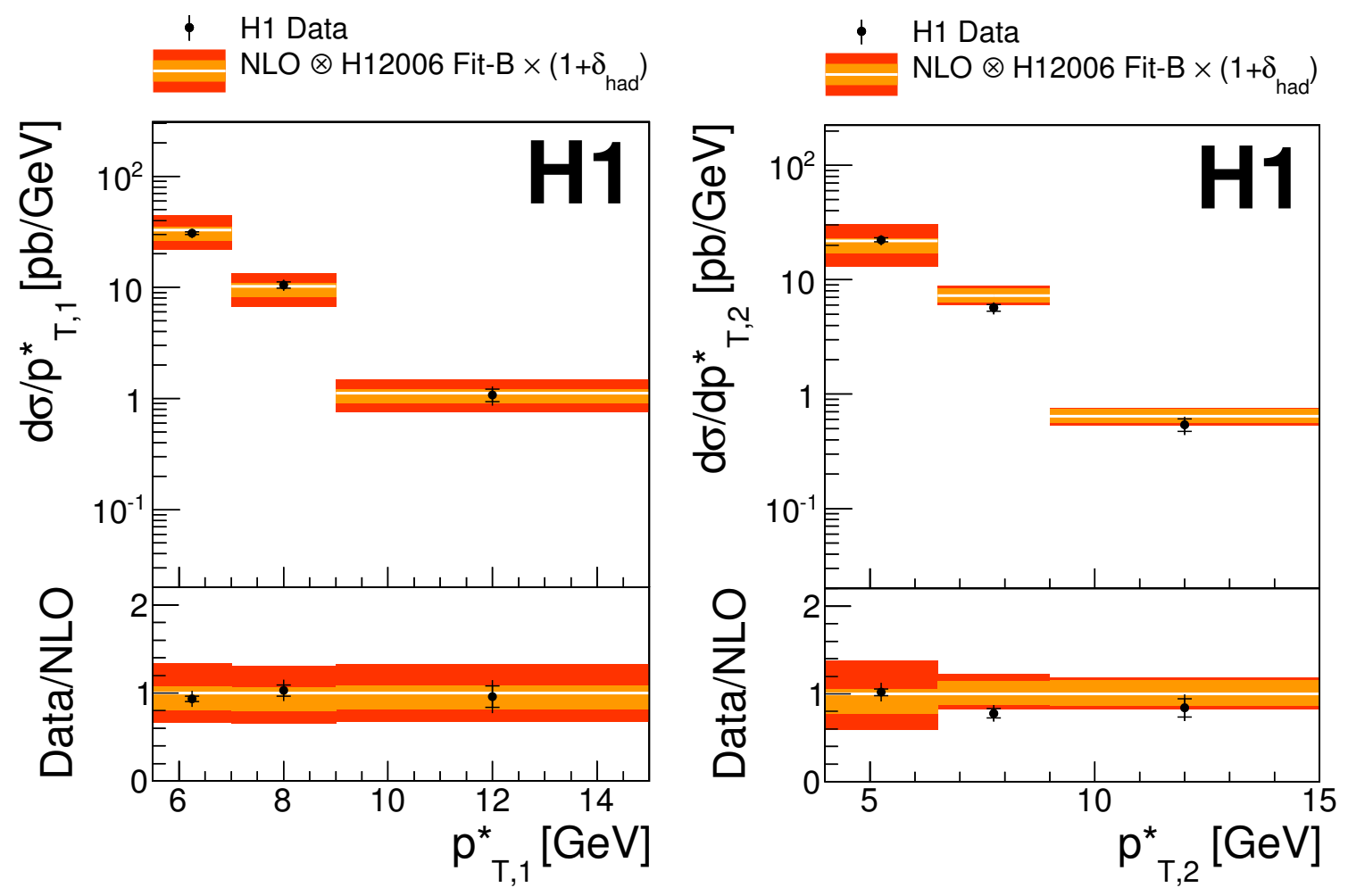

Figure 5: Diffractive dijet differential cross section as a function of $p_{\mathrm{T}, 1}^{*}$ and $p_{\mathrm{T}, 2}^{*}$. The inner error bars on the data points represent the statistical uncertainties, while the outer error bars include the systematic uncertainties added in quadrature. Further details are given in figure 3 . 

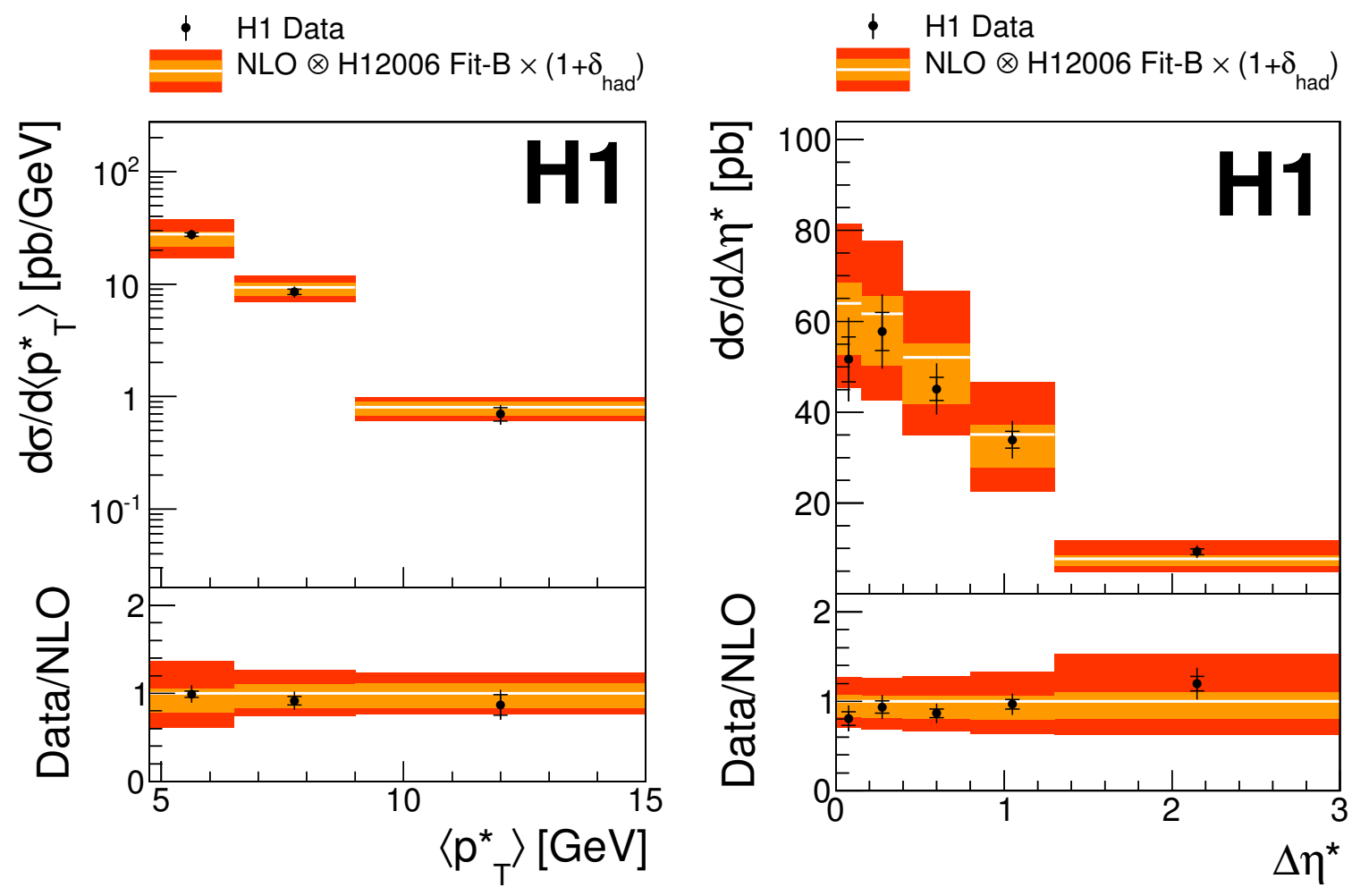

Figure 6: Diffractive dijet differential cross section as a function of $\left\langle p_{\mathrm{T}}^{*}\right\rangle$ and $\Delta \eta^{*}$. The inner error bars on the data points represent the statistical uncertainties, while the outer error bars include the systematic uncertainties added in quadrature. Further details are given in figure 3 . 


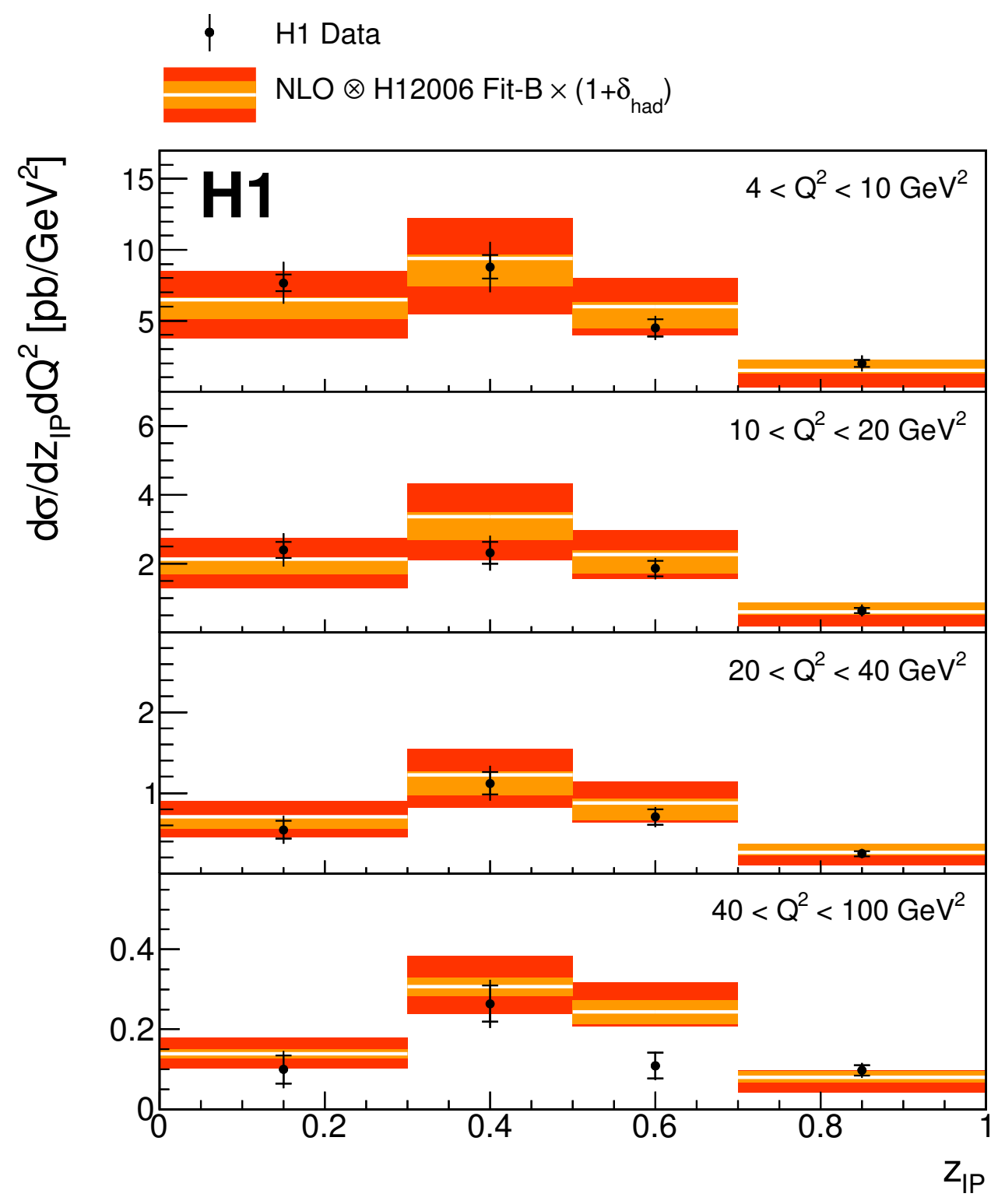

Figure 7: Double-differential cross section as a function of $z_{\mathbb{P}}$ and $Q^{2}$. The inner error bars on the data points represent the statistical uncertainties, while the outer error bars include the systematic uncertainties added in quadrature. Further details are given in figure 3 . 


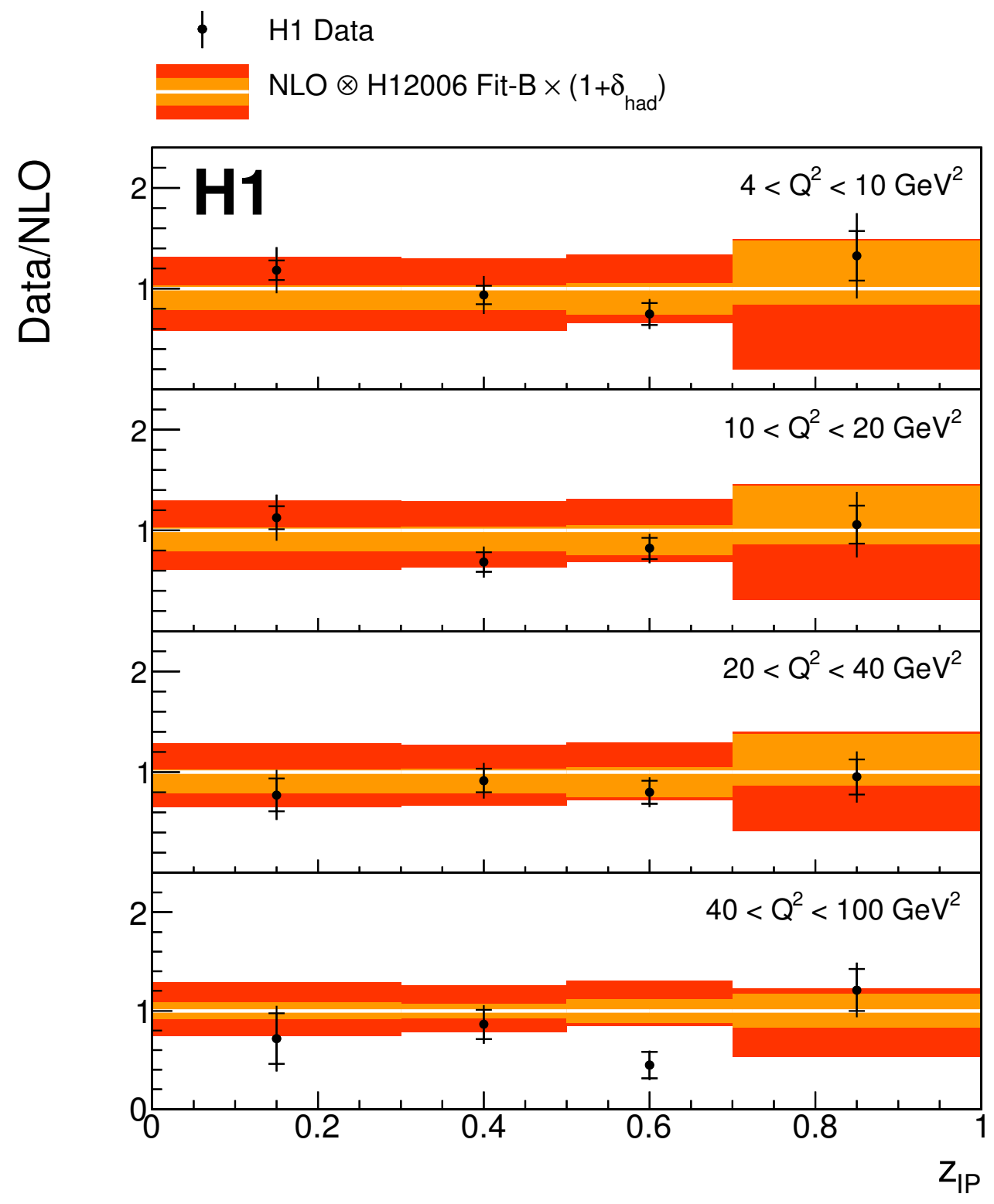

Figure 8: Ratio of the double-differential cross section to the NLO prediction as a function of $z_{\mathbb{P}}$ and $Q^{2}$. The inner error bars on the data points represent the statistical uncertainties, while the outer error bars include the systematic uncertainties added in quadrature. Further details are given in figure 3 . 


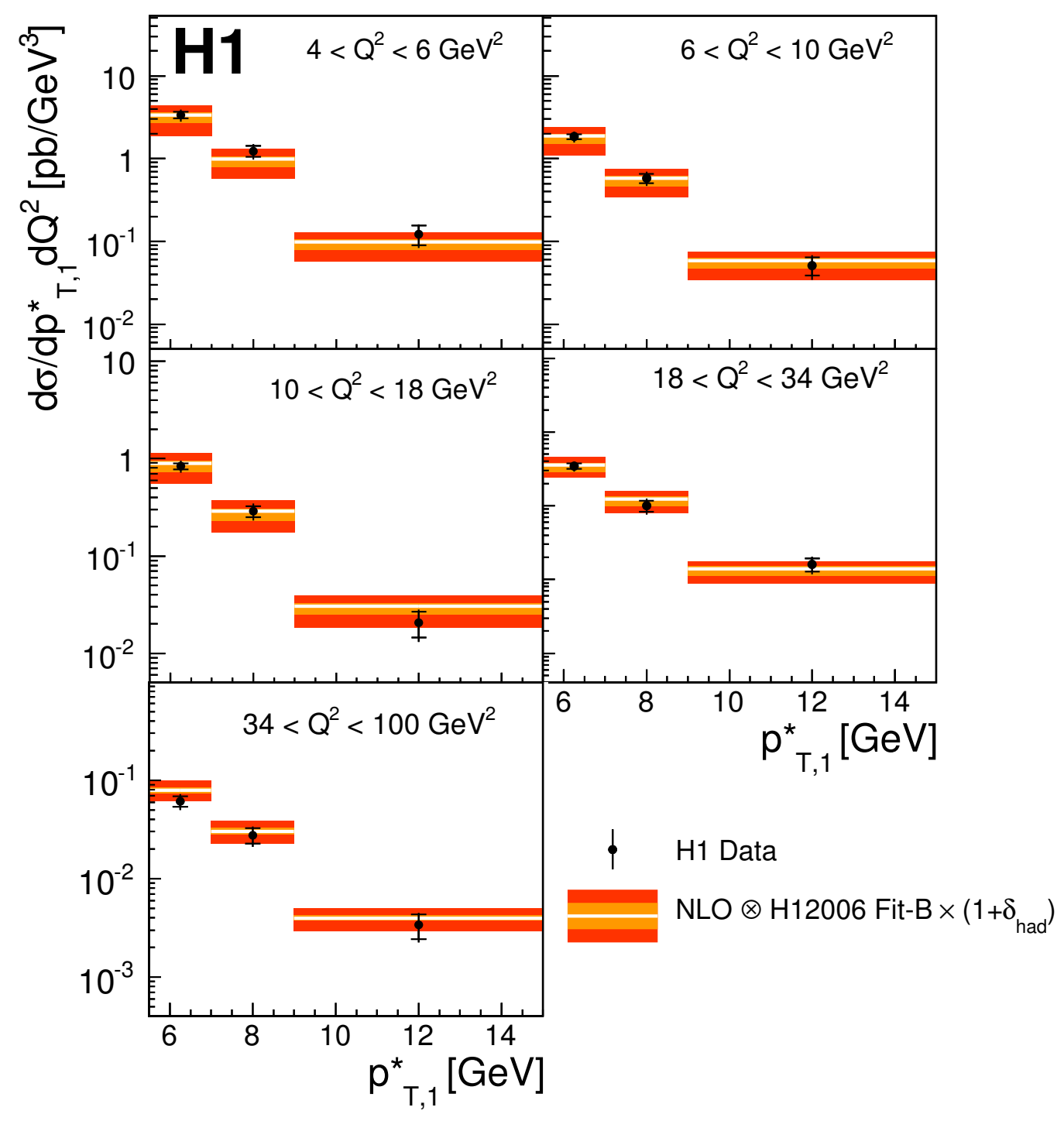

Figure 9: Double-differential cross section as a function of $p_{\mathrm{T}, 1}^{*}$ and $Q^{2}$. The inner error bars on the data points represent the statistical uncertainties, while the outer error bars include the systematic uncertainties added in quadrature. Further details are given in figure 3 . 


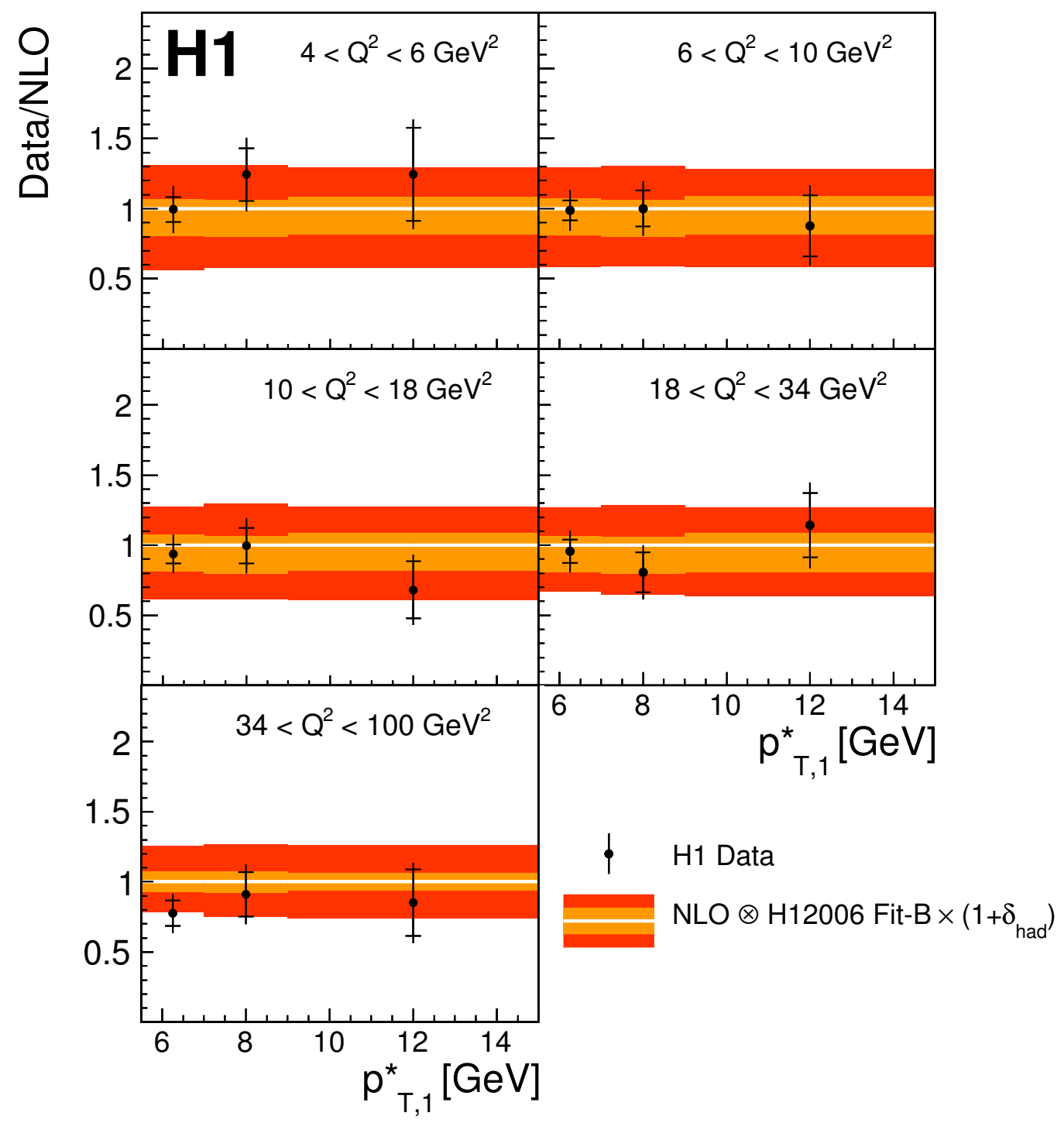

Figure 10: Ratio of the double-differential cross section to the NLO prediction as a function of $p_{\mathrm{T}, 1}^{*}$ and $Q^{2}$. The inner error bars on the data points represent the statistical uncertainties, while the outer error bars include the systematic uncertainties added in quadrature. Further details are given in figure 3. 
Open Access. This article is distributed under the terms of the Creative Commons Attribution License (CC-BY 4.0), which permits any use, distribution and reproduction in any medium, provided the original author(s) and source are credited.

\section{References}

[1] E. Feinberg and I. Pomeranchuk, High energy inelastic diffraction phenomena, Suppl. Nuovo Cim. 3 (1956) 652.

[2] K.A. Goulianos, Diffractive Interactions of Hadrons at High-Energies, Phys. Rept. 101 (1983) 169 [INSPIRE].

[3] L. Trentadue and G. Veneziano, Fracture functions: An improved description of inclusive hard processes in QCD, Phys. Lett. B 323 (1994) 201 [INSPIRE].

[4] H1 collaboration, A. Aktas et al., Measurement and QCD analysis of the diffractive deep-inelastic scattering cross-section at HERA, Eur. Phys. J. C 48 (2006) 715 [hep-ex/0606004] [INSPIRE].

[5] V.N. Gribov and L.N. Lipatov, Deep inelastic e p scattering in perturbation theory, Sov. J. Nucl. Phys. 15 (1972) 438 [inSPIRE].

[6] Y.L. Dokshitzer, Calculation of the Structure Functions for Deep Inelastic Scattering and $e^{+} e^{-}$Annihilation by Perturbation Theory in Quantum Chromodynamics., Sov. Phys. JETP 46 (1977) 641 [INSPIRE].

[7] G. Altarelli and G. Parisi, Asymptotic Freedom in Parton Language, Nucl. Phys. B 126 (1977) 298 [INSPIRE].

[8] J.C. Collins, Proof of factorization for diffractive hard scattering, Phys. Rev. D 57 (1998) 3051 [Erratum ibid. D 61 (2000) 019902] [hep-ph/9709499] [INSPIRE].

[9] G. Ingelman and P.E. Schlein, Jet Structure in High Mass Diffractive Scattering, Phys. Lett. B 152 (1985) 256 [INSPIRE].

[10] A. Donnachie and P.V. Landshoff, Diffractive Deep Inelastic Lepton Scattering, Phys. Lett. B 191 (1987) 309 [Erratum ibid. B 198 (1987) 590] [INSPIRE].

[11] ZEUS collaboration, S. Chekanov et al., Dissociation of virtual photons in events with a leading proton at HERA, Eur. Phys. J. C 38 (2004) 43 [hep-ex/0408009] [INSPIRE].

[12] H1 collaboration, C. Adloff et al., Diffractive jet production in deep inelastic $e^{+} p$ collisions at HERA, Eur. Phys. J. C 20 (2001) 29 [hep-ex/0012051] [InSPIRE].

[13] H1 collaboration, A. Aktas et al., Dijet Cross sections and Parton Densities in Diffractive DIS at HERA, JHEP 10 (2007) 042 [arXiv: 0708.3217] [INSPIRE].

[14] ZEUS collaboration, S. Chekanov et al., Dijet production in diffractive deep inelastic scattering at HERA, Eur. Phys. J. C 52 (2007) 813 [arXiv:0708.1415] [INSPIRE].

[15] H1 collaboration, F.D. Aaron et al., Measurement of Dijet Production in Diffractive Deep-Inelastic Scattering with a Leading Proton at HERA, Eur. Phys. J. C 72 (2012) 1970 [arXiv: 1111.0584] [INSPIRE].

[16] H. Jung, RAPGAP 31, Hard diffractive scattering in high-energy ep collisions and the Monte Carlo generator RAPGAP, Comput. Phys. Commun. 86 (1995) 147 [INSPIRE]. 
[17] M. Bengtsson and T. Sjöstrand, Parton Showers in Leptoproduction Events, Z. Phys. C 37 (1988) 465 [INSPIRE].

[18] B. Andersson, G. Gustafson, G. Ingelman and T. Sjöstrand, Parton Fragmentation and String Dynamics, Phys. Rept. 97 (1983) 31 [InSPIRE].

[19] T. Sjöstrand, High-energy physics event generation with PYTHIA 5.7 and JETSET 7.4, Comput. Phys. Commun. 82 (1994) 74 [INSPIRE].

[20] B. List and A. Mastroberardino, DIFFVM, in proceedings of the Monte Carlo Generators for HERA Physics Workshop 1998/99, A.T. Doyle, G. Grindhammer, G. Ingelman and H. Jung eds., Hamburg, Germany (1999) 396, [DESY-PROC-1999-02].

[21] H1 collaboration, C. Adloff et al., Measurement of dijet cross-sections at low $Q^{2}$ and the extraction of an effective parton density for the virtual photon, Eur. Phys. J. C 13 (2000) 397 [hep-ex/9812024] [INSPIRE].

[22] G.A. Schuler and T. Sjöstrand, Parton distributions of the virtual photon, Phys. Lett. B 376 (1996) 193 [hep-ph/9601282] [INSPIRE].

[23] A. Kwiatkowski, H. Spiesberger and H.J. Mohring, Heracles: An Event Generator for ep Interactions at HERA Energies Including Radiative Processes: Version 1.0, Comput. Phys. Commun. 69 (1992) 155 [INSPIRE].

[24] J. Pumplin et al., New generation of parton distributions with uncertainties from global QCD analysis, JHEP 07 (2002) 012 [hep-ph/0201195] [INSPIRE].

[25] R. Brun et al., GEANT-3, (1987), [CERN-DD/EE-84-1].

[26] Z. Nagy and Z. Trócsányi, Next-to-leading order calculation of four jet observables in electron positron annihilation, Phys. Rev. D 59 (1999) 014020 [Erratum ibid. D 62 (2000) 099902] [hep-ph/9806317] [INSPIRE].

[27] Z. Nagy and Z. Trócsányi, Multijet cross-sections in deep inelastic scattering at next-to-leading order, Phys. Rev. Lett. 87 (2001) 082001 [hep-ph/0104315] [INSPIRE].

[28] W. Giele et al., The QCD/SM working group: Summary report, in Proceedings of the Workshop on Physics at TeV Colliders (2001), Les Houches, France (2002) pg. 275 [hep-ph/0204316] [INSPIRE].

[29] H1 collaboration, C. Adloff et al., Measurement and QCD analysis of jet cross-sections in deep inelastic positron-proton collisions at $\sqrt{s}$ of $300 \mathrm{GeV}$, Eur. Phys. J. C 19 (2001) 289 [hep-ex/0010054] [INSPIRE].

[30] T. Kluge, K. Rabbertz and M. Wobisch, FastNLO: Fast $p Q C D$ calculations for PDF fits, In Proceedings of the 14th International Workshop on Deep Inelastic Scattering (DIS 2006), M. Kuze, K. Nagano and K. Tokushuku eds., Tsukuba, Japan (2007) pg. 483 [hep-ph/0609285] [INSPIRE].

[31] FastNLO collaboration, D. Britzger, K. Rabbertz, F. Stober and M. Wobisch, New features in version 2 of the fastNLO project, in proceedings of the 20th International Workshop on Deep-Inelastic Scattering and Related Subjects (DIS 2012), I. Brock ed., Bonn, Germany, (2012) pg. 217 [arXiv: 1208.3641] [INSPIRE].

[32] L. Lönnblad, ARIADNE version 4: A program for simulation of QCD cascades implementing the color dipole model, Comput. Phys. Commun. 71 (1992) 15 [INSPIRE]. 
[33] H1 collaboration, I. Abt et al., The H1 detector at HERA, Nucl. Instrum. Meth. A 386 (1997) 310 [INSPIRE].

[34] H1 Calorimeter Group collaboration, B. Andrieu et al., The H1 liquid argon calorimeter system, Nucl. Instrum. Meth. A 336 (1993) 460 [INSPIRE].

[35] H1 Calorimeter Group collaboration, B. Andrieu et al., Beam tests and calibration of the $H 1$ liquid argon calorimeter with electrons, Nucl. Instrum. Meth. A 350 (1994) 57 [INSPIRE].

[36] H1 Calorimeter Group collaboration, B. Andrieu et al., Results from pion calibration runs for the H1 liquid argon calorimeter and comparisons with simulations, Nucl. Instrum. Meth. A 336 (1993) 499 [INSPIRE].

[37] H1 SPACAL Group collaboration, R.D. Appuhn et al., The H1 lead/scintillating fiber calorimeter, Nucl. Instrum. Meth. A 386 (1997) 397 [INSPIRE].

[38] H1 SPACAL GRoup collaboration, R.D. Appuhn et al., Hadronic response and e/pi separation with the H1 lead/fiber calorimeter, Nucl. Instrum. Meth. A 382 (1996) 395 [INSPIRE].

[39] H1 collaboration, F.D. Aaron et al., Determination of the Integrated Luminosity at HERA using Elastic QED Compton Events, Eur. Phys. J. C 72 (2012) 2163 [Erratum ibid. C 74 (2012) 2733] [arXiv: 1205. 2448] [INSPIRE].

[40] H1 collaboration, C. Adloff et al., Inclusive measurement of diffractive deep inelastic ep scattering, Z. Phys. C 76 (1997) 613 [hep-ex/9708016] [INSPIRE].

[41] M. Peez, Search for deviations from the standard model in high transverse energy processes at the electron proton collider HERA (in French), Dissertation, Univ. Claude Bernard, Lyon France, DESY-THESIS-2003-023, CPPM-T-2003-04 (2003), available at http://www-h1.desy.de/psfiles/theses/h1th-317.pdf.

[42] S. Hellwig, Investigation of the $D^{*}-\pi_{\text {slow }}$ double tagging method for the analysis of charm (in German), Diploma Thesis, Hamburg University (2004), http://www-h1.desy.de/psfiles/theses/h1th-341.pdf.

[43] R. Kogler, Measurement of jet production in deep-inelastic ep scattering at HERA, Dissertation, Universität Hamburg, DESY-THESIS-2011-003, MPP-2010-175 (2010), http://www-h1.desy.de/psfiles/theses/h1th-590.pdf.

[44] S. Catani, Y.L. Dokshitzer and B.R. Webber, The $K^{-}$perpendicular clustering algorithm for jets in deep inelastic scattering and hadron collisions, Phys. Lett. B 285 (1992) 291 [INSPIRE].

[45] M. Cacciari and G.P. Salam, Dispelling the $N^{3}$ myth for the $k_{t}$ jet-finder, Phys. Lett. B 641 (2006) 57 [hep-ph/0512210] [INSPIRE].

[46] B. Pokorny, Measurement of Diffractive Dijet Production in Deep Inelastic Scattering at HERA Collider, Dissertation, Charles University in Prague (2014) http://www-h1.desy.de/psfiles/theses/h1th-850.pdf.

[47] S. Schmitt, TUnfold: an algorithm for correcting migration effects in high energy physics, 2012 JINST 7 T10003 [arXiv: 1205.6201] [INSPIRE].

[48] F.D. Aaron et al., Measurement of the cross section for diffractive deep-inelastic scattering with a leading proton at HERA, Eur. Phys. J. C 71 (2011) 1578 [arXiv:1010.1476] [INSPIRE]. 
[49] H1 collaboration, F.D. Aaron et al., Inclusive Measurement of Diffractive Deep-Inelastic Scattering at HERA, Eur. Phys. J. C 72 (2012) 2074 [arXiv: 1203.4495] [InSPIRE].

[50] H1 collaboration, V. Andreev et al., Measurement of Multijet Production in ep Collisions at High $Q^{2}$ and Determination of the Strong Coupling $\alpha_{s}$, arXiv:1406.4709 [INSPIRE].

[51] Particle Data Group collaboration, J. Beringer et al., Review of Particle Physics (RPP), Phys. Rev. D 86 (2012) 010001 [InSPIRE].

[52] S. Bethke, World Summary of $\alpha_{s}$ (2012), Nucl. Phys. Proc. Suppl. 234 (2013) 229 [arXiv: 1210.0325] [INSPIRE].

[53] H1 collaboration, F.D. Aaron et al., Jet Production in ep Collisions at Low $Q^{2}$ and Determination of $\alpha_{s}$, Eur. Phys. J. C 67 (2010) 1 [arXiv:0911.5678] [InSPIRE].

[54] ZEUS collaboration, H. Abramowicz et al., Inclusive-jet photoproduction at HERA and determination of alphas, Nucl. Phys. B 864 (2012) 1 [arXiv:1205.6153] [INSPIRE].

[55] D0 collaboration, V.M. Abazov et al., Determination of the strong coupling constant from the inclusive jet cross section in p p collisions at $\sqrt{s}=1.96 \mathrm{TeV}$, Phys. Rev. D 80 (2009) 111107 [arXiv: 0911.2710] [INSPIRE].

[56] D0 collaboration, V.M. Abazov et al., Measurement of angular correlations of jets at $\sqrt{s}=1.96 \mathrm{TeV}$ and determination of the strong coupling at high momentum transfers, Phys. Lett. B 718 (2012) 56 [arXiv:1207.4957] [INSPIRE].

[57] CMS collaboration, Measurement of the ratio of the inclusive 3-jet cross section to the inclusive 2-jet cross section in pp collisions at $\sqrt{s}=7 \mathrm{TeV}$ and first determination of the strong coupling constant in the TeV range, Eur. Phys. J. C 73 (2013) 2604 [arXiv: 1304.7498] [INSPIRE].

[58] CMS collaboration, Constraints on parton distribution functions and extraction of the strong coupling constant from the inclusive jet cross section in pp collisions at $\sqrt{s}=7 \mathrm{TeV}$, arXiv: 1410.6765 [INSPIRE]. 